\title{
Electromagnetic Model of a SPR Sensor Coupled to Array of Nanoparticles by Periodic Green's Function
}

\author{
André Cruz $\mathbb{D D}^{1},{ }^{1}$ Victor Dmitriev, ${ }^{1}$ Tommaso Del Rosso, ${ }^{2}$ and Karlo Costa ${ }^{1 D}{ }^{1}$ \\ ${ }^{1}$ Department of Electrical Engineering, Federal University of Pará, Belém-PA, Brazil \\ ${ }^{2}$ Department of Physics, Pontifical Catholic University of Rio de Janeiro, Rio de Janeiro-RJ, Brazil \\ Correspondence should be addressed to André Cruz; andcruz@ufpa.br
}

Received 28 March 2019; Accepted 3 June 2019; Published 16 July 2019

Guest Editor: Sandra Costanzo

Copyright (C) 2019 André Cruz et al. This is an open access article distributed under the Creative Commons Attribution License, which permits unrestricted use, distribution, and reproduction in any medium, provided the original work is properly cited.

\begin{abstract}
In this paper, we present a theoretical study of a Surface Plasmon Resonance Sensor in the Surface Plasmon Coupled Emission (SPCE) configuration. A periodic planar array of core-shell gold nanoparticles (AuNps), chemically functionalized to aggregate fluorescent molecules, is coupled to the sensor structure. These nanoparticles, characterized as target particles, are modeled as equivalent nanodipoles. The electromagnetic modeling of the device was performed using the spectral representation of the magnetic potential by Periodic Green's Function (PGF). Parametric results of spatial electric and magnetic fields are presented at wavelength $632.8 \mathrm{~nm}$. We also present a spectral analysis of the magnetic potential, where we verify the appearance of the surface plasmon polariton (SPP) waves. To validate the analytical method, we compared the limit case of small concentration of nanoparticles with published works. We also present a convergence analysis of the solution as a function of the concentration of nanoparticles in the periodic array. The results show that the theoretical method of PFG can be efficiently used as a tool for design of this sensing device.
\end{abstract}

\section{Introduction}

In the last two decades, the development of new optical devices based on metallic structures has been of great interest. This is due to the interesting optical and electromagnetic properties that the metals present in the regime of high frequency $[1,2]$. The interaction between metals and electromagnetic waves, in the optical regime, produces a collective oscillatory behavior in the gas of free electrons in the metal in phase opposite to the incident field, resulting in the formation of a surface wave with evanescent characteristic in the metal/dielectric interface, known as surface plasmon wave [3]. This phenomenon is related to the negative real part of the dielectric function of metal, which occurs when the excitation is due by optical fields $[3,4]$. In view of these properties, noble metals have been used for development of devices based on the Surface Plasmon Resonance (SPR), in planar structures, and Localized Surface Plasmon Resonance (LSPR), in isolated metallic nanoparticles [3-5].

Recently, research has shown that, on particular conditions, SPP waves can be efficiently used to control the near field intensification in metal nanostructures $[6,7]$. The study of these phenomena has been of great importance for the development of SPR sensors devices for applications in biosensing, photodetection, spectroscopy, and detection of metallic nanopoluents resulting from nanofabrication processes $[7,8]$. A conventional SPR sensor in the Surface Plasmon Coupled Emission (SPCE) configuration consists of a multilayer structure, generally a thin film of noble metal (gold or silver) over a dielectric prism, excited by an external laser source. On the structure of the device is coupled a microfluidic channel where, among other compounds, fluorophores are present, luminescent substances that when excited emit optical radiation [9-11]. In this configuration, it has been proposed to use chemically functionalized gold nanoparticles (AuNps) to obtain affinity with the luminescent substance, and then these AuNps couple to chemical binders on the surface of the sensor. The detection of high sensitivity is associated to the increase in the near field of nanoparticles that induce plasmon waves, amplifying the luminescence of the fluorophores and consequently, exciting SPP waves in the metal surface of the sensor [10-13]. Recently, chemical 
reactions in liquid solutions have been commonly used as a low cost procedure for AuNps production. In this process there is the presence of a surfactant agent that provides chemical and mechanical stability to the nanoparticle. The result is a type of metal particle covered with a thin superficial dielectric layer, which is called Core-shell. The optical response of these spherical particles in a free space was verified in [5].

In $[10,11]$, spatial and spectral analyses were performed for a single particle immobilized on the SPCE sensor structure, and from this, the radiated fields in the structure were verified. However, the existence of the electromagnetic interaction of more than one particle excited by the external source must be considered, yet, the modeling of the random spatial distribution of nanoparticles in a sample is still a great challenge. A coherent way to approximate this distribution is to consider that the sample takes the form of a periodic planar array of nanoparticles uniformly distributed over the gold layer [4].

The electromagnetic response of a multilayer structure excited by a planar array of nanosources can be described by the method of discrete spectral representation by Periodic Green's Function (PGF). However, when the concentration of AuNps becomes small, the discrete spectrum converges to a continuous spectrum, which is similar to that analyzed in $[10,11]$. A brief introduction about the analysis of this sensor by PGF is discussed in [14], where the authors presented only a spectral analysis of the dominant plasmonic mode in the sensor structure.

The objective of this work is to present a electromagnetic model of a surface plasmon resonance sensor in the SPCE configuration, coupled with a planar periodic array of CoreShell AuNps. For this sensor, we obtained a spectral representation of the magnetic vector potential by the Periodic Green's Function. To validate the method, we compared the limit case of low concentration of nanoparticles with the similar case analyzed in $[10,11]$; in addition, we verified the convergence of the method. Magnetic potential field and electromagnetic fields were analyzed by checking the effects of the thickness of the gold layer on the sensor structure, in addition to the effects of the dipole moment inclination induced on the nanoparticles. In this work, we expanded the spectral analysis to verify the effects of the plasmon poles on the spectral representation of the complex Fourier series.

\section{Functional Description of the SPCE Sensor}

The principle of operation of the SPCE sensor is based on the interaction of the field re-irradiated by the target particle on the sensor structure, exciting plasmon modes that creates polarized propagating waves at the sensor output [9-11]. As a target particle, it is proposed to use chemically-functionalized Core-Shell AuNps to attract fluorophores, enhancing the sensor's optical response. The functional illustration of the SPCE sensor is shown in Figure 1.

The physical structure of the sensor is formed by a gold layer deposited above a dielectric prism and below a microfluidic channel where multicompounds, fluorophores, and Core-Shell AuNps are present in suspension. On the

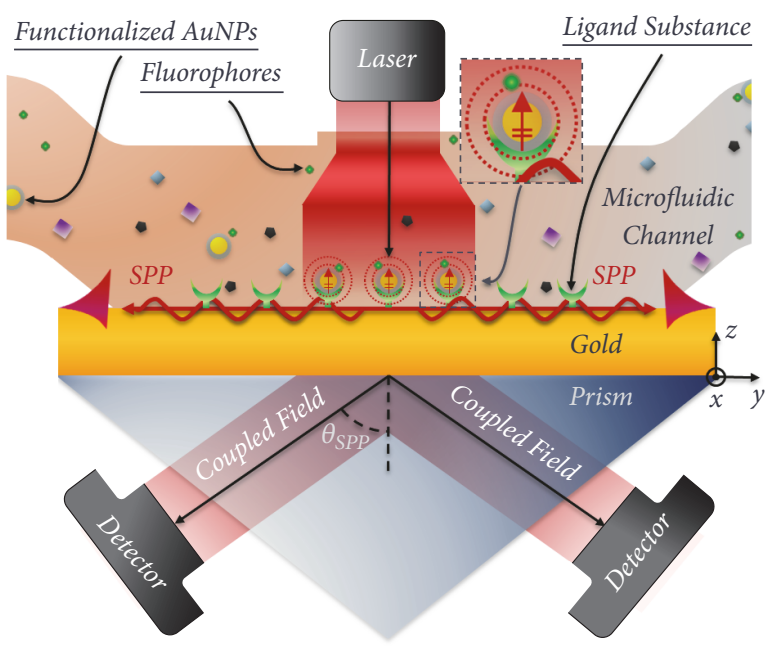

FIGURE 1: Functional illustration of the SPCE sensor coupled to a microfluidic channel.

gold layer there is a binding substance with gold/target particle affinity that immobilizes the AuNps aggregated with fluorophores on the surface of the sensor. This substance, considered to be electrically inert, is used as a chemical spacer and acts to separate the immobilized particles and the gold sheet.

The excitation, realized by a monochromatic optical laser with wavelength $\lambda=632.8 \mathrm{~nm}$, is directly applied to the sample above the gold layer, exciting the target particles, which consequently re-irradiate fields that excite SPP waves in the interface between the gold layer and the dielectric of the microfluidic channel. These SPP waves are excited by the TM evanescent radiation component of the excited particle. Thus, part of the plasmon wave is transmitted through the gold layer, coupling in the region of the prism a transversal magnetic wave as a function of the SPP wave. The formation of this TM wave provides a far field pattern like a light cone in the prism region, where $\theta_{S P P}$ is the characteristic plasmonic angle of this cone (Figure 1).

Although the radiation scattered by the target particle is not polarized, the field that mates in the region of the prism is highly polarized in TM. This characteristic can be understood by the opposite process, which occurs in the excitation of SPP waves by plane waves, exclusively polarized in TM, as in the Kretschmann configuration. For this reason, the SPCE sensor acts as a natural filter for TM polarization.

Depending on the electromagnetic characteristics of the sample formed by the multicompounds, in other words, the dielectric characteristics of the environment formed by the medium and multicompounds, in the microfluidic channel, the intensity, geometry, and angle $\theta_{S P P}$ of the light cone, measured by a detector, are changed, thus defining the output of the sensor.

\section{Electromagnetic Model of Sensor}

3.1. Description of the Problem. The electromagnetic interaction between the laser source and the Core-Shell AuNps 


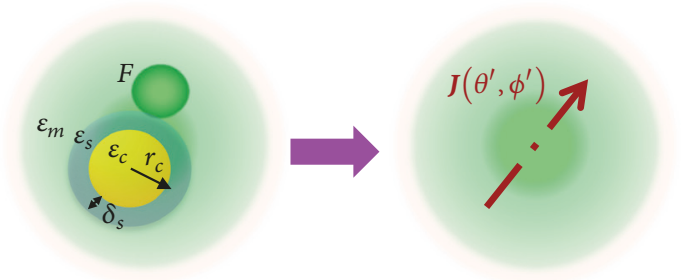

Figure 2: Equivalent between excited target particle and hertzian dipole.

can be described by the quasi-static Rayleigh scattering, since the wavelength of the source is much larger than the particle dimensions [5]; thus the radiation scattered by the nanoparticle is described by the fundamental dipole moment $p_{c s}=\alpha_{c s} E_{0}$, characterized by polarizability (1), excited by electric field $E_{0}$.

$$
\begin{aligned}
\alpha_{c s} & =4 \pi \varepsilon_{m}\left(r_{c}+\delta_{s}\right)^{3} \\
& \cdot\left[\frac{f\left(\varepsilon_{c}-\varepsilon_{m}\right)\left(2 \varepsilon_{s}+\varepsilon_{m}\right)+\left(2 \varepsilon_{s}+\varepsilon_{c}\right)\left(\varepsilon_{s}-\varepsilon_{m}\right)}{f\left(\varepsilon_{c}-\varepsilon_{m}\right)\left(2 \varepsilon_{s}-2 \varepsilon_{m}\right)+\left(2 \varepsilon_{s}+\varepsilon_{c}\right)\left(\varepsilon_{s}+2 \varepsilon_{m}\right)}\right]
\end{aligned}
$$

with $f=r_{c}^{3} /\left(r_{c}+\delta_{s}\right)^{3}$ being the fraction of the total volume of the particle occupied by the core, $r_{c}$ and $\delta_{s}$ the radius of the core and the thickness of the nanoparticle shell, respectively, and $\varepsilon_{c}, \varepsilon_{s}$, and $\varepsilon_{m}$ the permittivities of the core, shell, and medium where the nanoparticles are inserted. Similarly, the spectral emission of the fluorescence for the excitation wavelength can be modeled by the polarizability of the fluorophore $\alpha_{F F}$ [15].

Due to the dimensions of the Core-Shell AuNps and the fluorophores, we can characterize the target particle excited by an effective dipole moment:

$$
p_{e f f}=p_{c s}+\alpha_{F F} R_{\mathrm{e}}^{F F}
$$

where $R_{e}^{F F}$ is the field reaction to the excitation of the fluorophore, which is a function of the excitation intensity and the Fluorescence $F$. From (2), target particles excited by the external (suppressed) source are modeled as sources of concentrated electric current $J$ (3) (hertzian dipoles), with current moments $I_{0} l=j \omega p_{\text {eff }}$ in the direction $\widehat{a}_{r}$ oriented by the parametric elevation $\theta^{\prime}$ and azimuth $\phi^{\prime}$ angles. The equivalence is shown in Figure 2.

$$
\mathbf{J}\left(\theta^{\prime}, \phi^{\prime}\right)=j \omega p_{e f f} \delta(x) \delta(y) \delta(z-h) \widehat{a}_{r}\left(\theta^{\prime}, \phi^{\prime}\right)
$$

In the microfluidic channel, the target particles are distributed randomly in space. Due to the fact that these are immobilized near the surface of the sensor, and the low concentration, we can approximate the sample as a planar array of uniformly distributed nanodipoles, equally distant from the gold sheet.

Since the sample was modeled as a periodic planar array, we can perform the field analysis by defining a particular cell with width $2 a$ at $x, 2 b$ at $y$, and with the current source located at a height $h$ by the chemical spacer (Figure 3 ).
The analysis region is delimited by three volumes $V 1, V 2$, and $V 3$; medium 1 , medium 2 , and medium 3 , respectively, are enclosed by the closed surfaces $S_{1}=S_{11}+S_{12}+S_{13}+$ $S_{14}+S_{15}+S_{16} ; S_{2}=S_{21}+S_{22}+S_{23}+S_{24}+S_{25}+S_{26}$; and $S_{3}=S_{31}+S_{32}+S_{33}+S_{34}+S_{35}+S_{36}$, thus forming three six-sided prisms (Figure 4).

The electric and magnetic fields were determined by the magnetic potential method, defined by the solution of the Helmholtz equation in the three media [16]:

$$
\begin{aligned}
-\left(\nabla^{2}+k_{1}^{2}\right) \mathbf{A}_{1} & =\mu_{1} \mathbf{J} \\
-\left(\nabla^{2}+k_{2,3}^{2}\right) \mathbf{A}_{2,3} & =0
\end{aligned}
$$

In order for the electromagnetic field to obey the periodic conditions, the potential field on surfaces $S_{v 1}, S_{v 2}, S_{v 3}, S_{v 4}$ must satisfy the boundary conditions (5) in the medium $v=1$, 2 and 3 . In regions distant from the source, the potential field must satisfy the limit boundary conditions (6).

$$
\begin{aligned}
\left.\mathbf{A}_{[v]}(a, y, z)\right|_{S_{[v] 1}} & =\left.\mathbf{A}_{[v]}(-a, y, z)\right|_{S_{[v] 2}}, \\
\left.\frac{\partial \mathbf{A}_{[v]}}{\partial x}(a, y, z)\right|_{S_{[v] 1}} & =\left.\frac{\partial \mathbf{A}_{[v]}}{\partial x}(-a, y, z)\right|_{S_{[v] 2}} \\
\left.\mathbf{A}_{[v]}(x, b, z)\right|_{S_{[v] 3}} & =\left.\mathbf{A}_{[v]}(x,-b, z)\right|_{S_{[v] 4}}, \\
\left.\frac{\partial \mathbf{A}_{[v]}}{\partial y}(x, b, z)\right|_{S_{[v] 3}} & =\left.\frac{\partial \mathbf{A}_{[v]}}{\partial y}(x,-b, z)\right|_{S_{[v] 4}} \\
\left.\lim _{z \longrightarrow+\infty} \mathbf{A}_{1}(x, y, z)\right|_{S_{15}} & =0, \\
\left.\lim _{z \longrightarrow-\infty} \mathbf{A}_{3}(x, y, z)\right|_{S_{36}} & =0
\end{aligned}
$$

At the interfaces $z=d_{u}(u=1,2)$, formed by the surfaces $S_{u 6}$ and $S_{[u+1] 5}$, the potential field must obey (7) and (8), so that the conditions of continuity of tangential fields and normal flows are met.

$$
\begin{aligned}
& \left.A_{x_{[u+1]}, y_{[u+1]}}\right|_{z=d_{[u]}}=\left.A_{x_{[u]}, y_{[u]}}\right|_{z=d_{[u]}} \\
& \frac{1}{\mu_{[u+1]}} \frac{\partial}{\partial z}\left[\left.A_{\left.x_{[u+1]}, y_{[u+1]}\right]}\right|_{z=d_{[u]}}\right. \\
& =\frac{1}{\mu_{[u]}} \frac{\partial}{\partial z}\left[\left.A_{\left.x_{[u]}, y_{[u]}\right]}\right|_{z=d_{[u]}}=\left.\frac{1}{\mu_{[u]}} A_{z_{[u]}}\right|_{z=d_{[u]}}\right. \\
& \left.\left.\frac{1}{\mu_{[u+1]}} A_{z_{[u+1]}}\right|_{z=d_{[u]}}\left(\nabla \cdot \mathbf{A}_{[u+1]}\right)\right|_{z=d_{[u]}} \\
& \left.\frac{1}{\mu_{[u+1]} \varepsilon_{[u+1]}}\right|_{z=d_{[u]}}
\end{aligned}
$$

From the Partial Differential Equation (PDE) defined in (4) and the conditions in (5)-(8), the fields were determined 


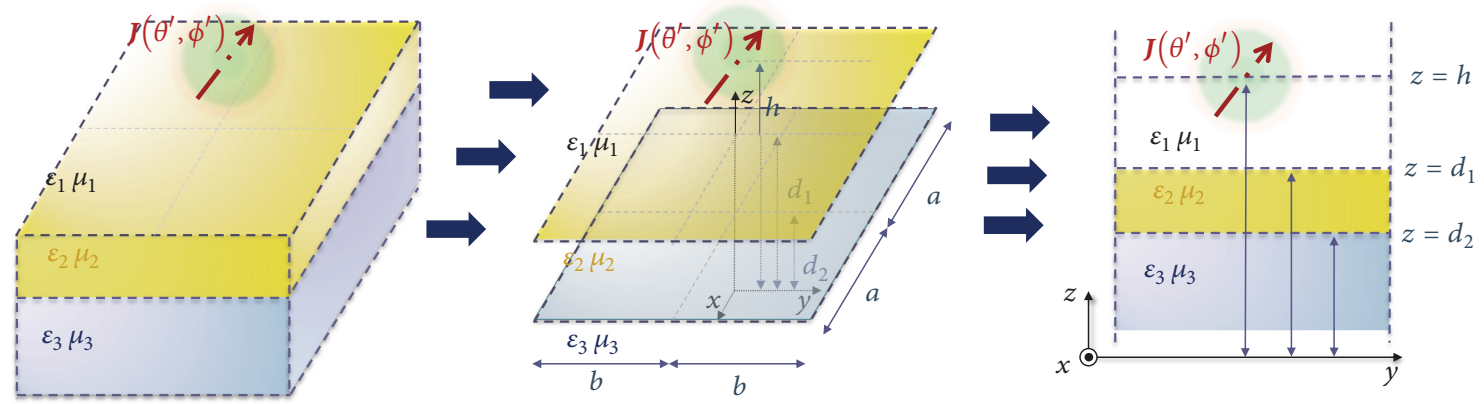

FIGURE 3: Definition of the analysis cell. (Left) 3D view of the three layers (microfluidic region (1), gold layer (2), and prism (3)) and one point dipole. (Middle) 3D view of the interfaces and geometry of the unit cell. (Right) Side view of the unit cell.

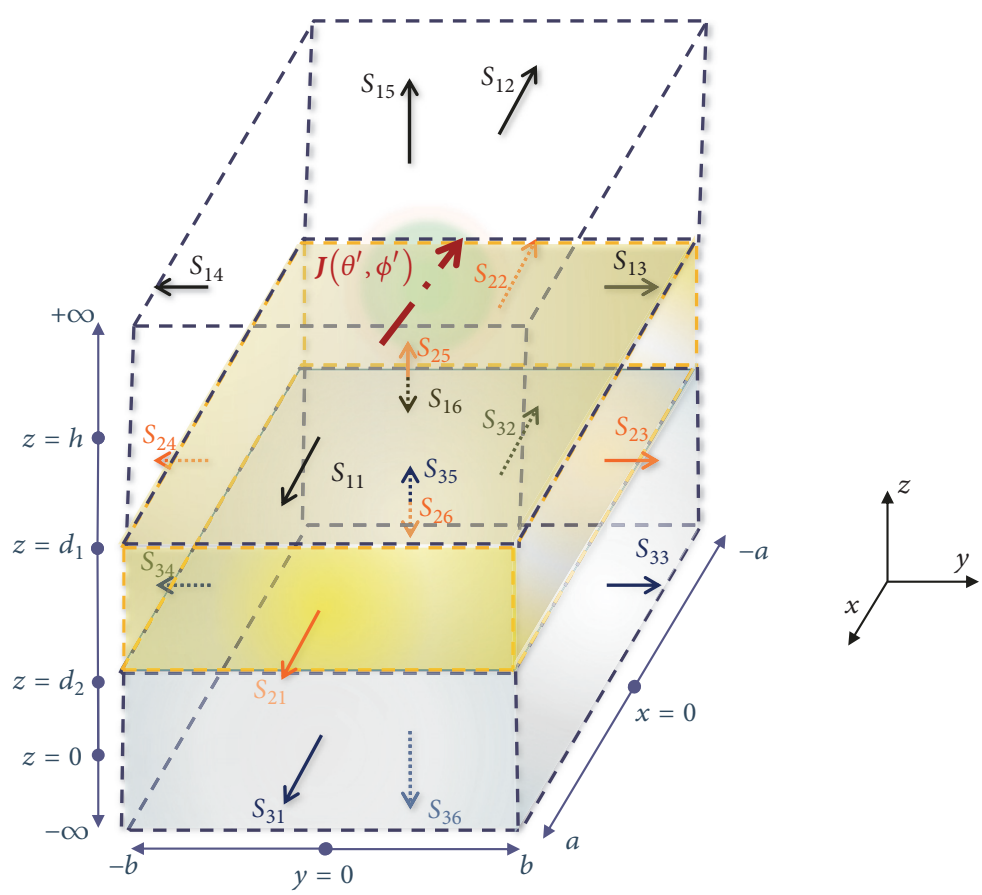

FIgURE 4: Analysis volume.

by the Periodic Green's Function in $x y$, with Limit and Neumann boundary conditions in $z$.

3.2. Magnetic Potential Tensor by PGF Method. The Periodic Green's Function is written in terms of the discrete spectral expansion in $x y$ (9), defined by the inverse transform of the complex Fourier series, applied to one-dimensional Green's function problem at $\mathrm{z}$ in the media 1,2 , and 3 [16].

$$
g_{1,2,3}\left(\mathbf{r}, \mathbf{r}^{\prime}\right)=\sum_{m=-\infty}^{\infty} \sum_{n=-\infty}^{\infty} g_{1,2,3_{m n}}\left(z, \mathbf{r}^{\prime}\right) u_{m}(x) u_{n}(y)
$$

$$
\begin{aligned}
& u_{m}(x)=\frac{1}{\sqrt{2 a}} e^{j k_{x} x} \\
& u_{n}(y)=\frac{1}{\sqrt{2 b}} e^{j k_{y} y}
\end{aligned}
$$

where $u_{m}(x)$ and $u_{n}(y)$ are the eigenfunctions of the periodic problem at $x$ and $y$, with eigenvalues $k_{x}=m \pi / a$ and $k_{y}=n \pi / b$. In the spectral domain $m n$, the one-dimensional Green's functions $z$ in the media 1, 2, and 3 are, respectively, $g_{1_{m n}}$ in $\left(z>d_{1}\right), g_{2_{m n}}$ in $\left(d_{1} \geq z \geq d_{2}\right)$, and $g_{3_{m n}}$ in $\left(z<d_{2}\right)$. 


$$
\begin{aligned}
& g_{1_{m n}}\left(z, \mathbf{r}^{\prime}\right)=\frac{1}{2 j k_{z 1}}\left[e^{-j k_{z 1}\left|z-z^{\prime}\right|}+e^{-j k_{z 1}\left(z+z^{\prime}-2 d_{1}\right)}\right] \bar{u}_{m}\left(x^{\prime}\right) \bar{u}_{n}\left(y^{\prime}\right) \\
& g_{2_{m n}}\left(z, \mathbf{r}^{\prime}\right)=\left[\frac{\cos k_{z 2}\left(\left|z-z^{\prime}\right|+\left[d_{2}-d_{1}\right]\right)+\cos k_{z 2}\left(\left[z^{\prime}+z\right]-\left[d_{2}+d_{1}\right]\right)}{2 k_{z 2} \sin k_{z 2}\left(d_{2}-d_{1}\right)}\right] \bar{u}_{m}\left(x^{\prime}\right) \bar{u}_{n}\left(y^{\prime}\right) \\
& g_{3_{m n}}\left(z, \mathbf{r}^{\prime}\right)=\frac{1}{2 j k_{z 3}}\left[e^{-j k_{z 3}\left|z^{\prime}-z\right|}+e^{j k_{z 3}\left(z+z^{\prime}-2 d_{2}\right)}\right] \bar{u}_{m}\left(x^{\prime}\right) \bar{u}_{n}\left(y^{\prime}\right)
\end{aligned}
$$

where $k_{z v}$ is the $\mathrm{z}$-propagation constant in the medium $v=$ $1,2,3$.

$$
k_{z[v]}=\sqrt{k_{[v]}^{2}-k_{x}^{2}-k_{y}^{2}}
$$

Applying the Green identity in each medium, using the boundary conditions defined in (5)-(8) and solving the system of equations, the magnetic potential field in the tensor form is given by [16]:

$$
\begin{aligned}
& \mathbf{A}_{1,2,3}(\mathbf{r}) \\
& =\sum_{m=-\infty}^{\infty} \sum_{n=-\infty}^{\infty}\left[\begin{array}{ccc}
A_{x x_{1,2,3}}^{m n} & 0 & 0 \\
0 & A_{y y_{1,2,3}}^{m n} & 0 \\
-j k_{x} A_{z x_{1,2,3}}^{m n} & -j k_{y} A_{z y_{1,2,3}}^{m n} & A_{z z_{1,2,3}}^{m n}
\end{array}\right] \\
& \cdot \mathbf{I}\left(\theta^{\prime}, \phi^{\prime}\right) e^{-j\left[k_{x} x+k_{y} y\right]}
\end{aligned}
$$

the element $A_{i j}$ being the component $i$, excited by the component $j$ of the current density per cell $\mathbf{I}$.

$$
\begin{aligned}
& \mathbf{I}\left(\theta^{\prime}, \phi^{\prime}\right)
\end{aligned}
$$

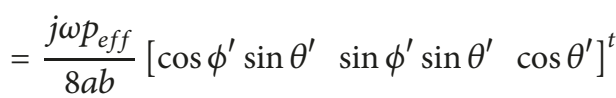

The elements of the magnetic potential tensor (16), in the $m n$ spectral domain and $z$ spatial domain, are, in medium 1 ,

$$
\begin{aligned}
A_{x x_{1}}^{m n}(z) & =A_{y y_{1}}^{m n}(z) \\
& =-j \mu_{1} \frac{1}{k_{z 1}}\left[e^{-j k_{z 1}|z-h|}+\widetilde{R}_{12}^{T E} e^{-j k_{z 1}\left(z+h-2 d_{1}\right)}\right] \\
A_{z x_{1}}^{m n}(z) & =A_{z y_{1}}^{m n}(z)=-\mu_{1} S_{1 d 1}^{E M} \frac{1}{k_{z 1}} e^{-j k_{z 1}\left(z+h-2 d_{1}\right)} \\
A_{z z_{1}}^{m n}(z) & =-j \mu_{1} \frac{1}{k_{z 1}}\left[e^{-j k_{z 1}|z-h|}+\widetilde{R}_{12}^{T M} e^{-j k_{z 1}\left(z+h-2 d_{1}\right)}\right]
\end{aligned}
$$

In medium 2,

$$
\begin{aligned}
& A_{x x_{2}}^{m n}(z)=A_{y y_{2}}^{m n}(z)=-j \mu_{2} \\
& \cdot \frac{1}{k_{z 2}}\left[e^{j k_{z 2}\left(z-d_{2}\right)}+R_{23}^{T E} e^{-j k_{z 2}\left(z-d_{2}\right)}\right] \frac{T_{23}^{T E}}{T_{23}^{T E}} e^{-j k_{z 1}\left(h-d_{1}\right)} \\
& A_{z x_{2}}^{m n}(z)=A_{z y_{2}}^{m n}(z)=-\mu_{2} \\
& \cdot \frac{1}{k_{z 2}}\left[S_{2 d 1}^{E M} e^{j k_{z 2}\left(z-d_{2}\right)}+S_{2 d 2}^{E M} e^{-j k_{z 2}\left(z-d_{2}\right)}\right] \\
& \cdot \widetilde{T}_{23}^{T M} e^{-j k_{z 1}\left(h-d_{1}\right)} \\
& A_{z z_{2}}^{m n}(z)=-j \mu_{2} \frac{1}{k_{z 2}}\left[e^{j k_{z 2}\left(z-d_{2}\right)}+R_{23}^{T M} e^{-j k_{z 2}\left(z-d_{2}\right)}\right] \frac{\varepsilon_{2}}{\varepsilon_{1}} \\
& \cdot \frac{\widetilde{T}_{23}^{T M}}{T_{23}^{T M}} e^{-j k_{z 1}\left(h-d_{1}\right)}
\end{aligned}
$$

In medium 3,

$$
\begin{aligned}
\mathrm{A}_{x x_{3}}^{m n}(z) & =A_{y y_{3}}^{m n}(z) \\
& =-j \mu_{3} \frac{1}{k_{z 3}} e^{j\left[k_{z 3}\left(z-d_{2}\right)-k_{z 1}\left(h-d_{1}\right)\right]} \frac{\mu_{2} k_{z 3}}{\mu_{3} k_{z 2}} \widetilde{T}_{23}^{T E} \\
A_{z x_{3}}^{m n}(z) & =A_{z y_{3}}^{m n}(z)=-\mu_{3} \frac{1}{k_{z 3}} e^{j\left[k_{z 3}\left(z-d_{2}\right)-k_{z 1}\left(h-d_{1}\right)\right]} S_{3 d 2}^{E M} \\
A_{z z_{3}}^{m n}(z) & =-j \mu_{3} \frac{1}{k_{z 3}} e^{j\left[k_{z 3}\left(z-d_{2}\right)-k_{z 1}\left(h-d_{1}\right)\right]} \frac{\varepsilon_{2} k_{z 3}}{\varepsilon_{1} k_{z 2}} \widetilde{T}_{23}^{T M}
\end{aligned}
$$

The Fresnel transmission and reflection coefficients of the TE and TM modes follow the definition [17]:

$$
\begin{aligned}
& T_{a b}^{T E}=1+R_{a b}^{T E}=1+\frac{\mu_{b} k_{z a}-\mu_{a} k_{z b}}{\mu_{b} k_{z a}+\mu_{a} k_{z b}} \\
& T_{a b}^{T M}=1+R_{a b}^{T M}=1+\frac{\varepsilon_{b} k_{z a}-\varepsilon_{a} k_{z b}}{\varepsilon_{b} k_{z a}+\varepsilon_{a} k_{z b}}
\end{aligned}
$$

The generalized reflection and transmission coefficients TE and TM are defined in (29)-(32).

$$
\widetilde{R}_{12}^{T E}=1-\frac{T_{21}^{T E}\left[1-R_{23}^{T E} e^{2 j k_{z 2}\left(d_{2}-d_{1}\right)}\right]}{\left[1-R_{21}^{T E} R_{23}^{T E} e^{2 j k_{z 2}\left(d_{2}-d_{1}\right)}\right]}
$$




$$
\begin{aligned}
\widetilde{T}_{23}^{T E} & =\frac{T_{21}^{T E} T_{23}^{T E} e^{j k_{z 2}\left(d_{2}-d_{1}\right)}}{\left[1-R_{21}^{T E} R_{23}^{T E} e^{2 j k_{z 2}\left(d_{2}-d_{1}\right)}\right]} \\
\widetilde{R}_{u 2}^{T M} & =1-\frac{T_{2 u}^{T M}\left[1-R_{2 u}^{T M} e^{2 j k_{z 2}\left(d_{2}-d_{1}\right)}\right]}{\left[1-R_{2 u}^{T M} R_{2 u}^{T M} e^{2 j k_{z 2}\left(d_{2}-d_{1}\right)}\right]}, \quad u=1,3 \\
\widetilde{T}_{23}^{T M} & =\frac{T_{21}^{T M} T_{23}^{T M} e^{j k_{z 2}\left(d_{2}-d_{1}\right)}}{\left[1-R_{21}^{T M} R_{23}^{T M} e^{2 j k_{z 2}\left(d_{2}-d_{1}\right)}\right]}
\end{aligned}
$$

The $S^{E M}$ coupling coefficients relate the reflection and transmission coefficients of TE and TM modes:

$$
\begin{aligned}
& S_{1 d 1}^{E M}=\frac{1}{2 k_{z 2}}\left[\zeta_{12} \frac{\varepsilon_{1} k_{z 2}}{\varepsilon_{2} k_{z 1}} \frac{\mu_{1}}{\mu_{2}}\left[1+\widetilde{R}_{12}^{T M}\right]\left[1+\widetilde{R}_{12}^{T E}\right]\right. \\
& \left.\quad+\zeta_{23} \frac{\varepsilon_{2} k_{z 1}}{\varepsilon_{3} k_{z 2}} \frac{\mu_{2}}{\mu_{3}} \widetilde{T}_{23}^{T M} \widetilde{T}_{23}^{T E}\right] \\
& S_{2 d 1}^{E M}=\frac{1}{2}\left[\zeta_{12} \frac{1}{k_{z 1}} \frac{\mu_{1}}{\mu_{2}} \frac{1}{T_{23}^{T M}}\left[1+\widetilde{R}_{12}^{T E}\right]\right. \\
& \left.+\zeta_{23} \frac{1}{k_{z 2}} \frac{\varepsilon_{2} \mu_{2}}{\varepsilon_{3} \mu_{3}} \frac{R_{21}^{T M}}{T_{21}^{T M}} \widetilde{T}_{23}^{T E} e^{j k_{z 2}\left(d_{2}-d_{1}\right)}\right] \\
& S_{2 d 2}^{E M}=\frac{1}{2}\left[\zeta_{12} \frac{1}{k_{z 1}} \frac{\mu_{1}}{\mu_{2}} \frac{R_{23}^{T M}}{T_{23}^{T M}}\left[1+\widetilde{R}_{12}^{T E}\right]\right. \\
& \left.+\zeta_{23} \frac{1}{k_{z 2}} \frac{\varepsilon_{2} \mu_{2}}{\varepsilon_{3} \mu_{3}} \frac{1}{T_{21}^{T M}} \widetilde{T}_{23}^{T E} e^{-j k_{z 2}\left(d_{2}-d_{1}\right)}\right] \\
& \left.\quad+\zeta_{23} \frac{\mu_{2}}{\mu_{3}}\left[1+\widetilde{R}_{32}^{T M}\right] \widetilde{T}_{23}^{T E}\right] \\
& S_{3 d 2}^{E M}=\frac{1}{2 k_{z 2}}\left[\zeta_{12} \frac{\mu_{1} k_{z 3}}{\mu_{2} k_{z 1}} \widetilde{T}_{23}^{T M}\left[1+\widetilde{R}_{12}^{T E}\right]\right.
\end{aligned}
$$

being $\zeta$ coupling parameters at interfaces $d_{1}$ and $d_{2}$ :

$$
\zeta_{a b}=\left[\frac{\varepsilon_{b} \mu_{b}-\varepsilon_{a} \mu_{a}}{\varepsilon_{a} \mu_{a}}\right]
$$

Given the magnetic potential, the magnetic and electric fields can be easily obtained through differential operations [18].

$$
\begin{aligned}
& \mathbf{H}=\frac{1}{\mu} \nabla \times \mathbf{A} \\
& \left.\mathbf{E}=\frac{1}{j \omega \mu \varepsilon} \nabla(\nabla \cdot \mathbf{A})\right)-j \omega \mathbf{A}
\end{aligned}
$$

3.3. Alternative Form of PGF Spectral Representation. The discrete spectral representations in the solutions of the PGF in (16) are done as double sums of $-\infty$ to $+\infty$, which may require a reasonable computational cost in the simulations. To reduce such cost, it is proposed to change the base functions in the sums using the Euler identity, provided that the spectral terms in (16) obey the following conditions:

$$
\begin{aligned}
& c_{m, n}(z)=c_{-m,-n}(z)=c_{m,-n}(z)=c_{-m, n}(z), \\
& c_{m, 0}(z)=c_{-m, 0}(z), \\
& c_{0, n}(z)=c_{0,-n}(z)
\end{aligned}
$$

That is, these spectral terms are even functions with the variables $m$ and $n$. The proposed identities are

$$
\begin{aligned}
S(z) & =\sum_{m=-\infty}^{\infty} \sum_{m=-\infty}^{\infty} c_{m n}(z) e^{-j\left[k_{x} x+k_{y} y\right]} \\
& =\sum_{m=0}^{\infty} \sum_{m=0}^{\infty} \epsilon_{m n} c_{m n}(z) \cos \left[k_{x} x\right] \cos \left[k_{y} y\right] \\
\frac{\partial S}{\partial x}(z) & =\sum_{m=-\infty}^{\infty} \sum_{m=-\infty}^{\infty} c_{m n}(z)\left[-j k_{x}\right] e^{-j\left[k_{x} x+k_{y} y\right]} \\
& =\sum_{m=0}^{\infty} \sum_{m=0}^{\infty} \epsilon_{m n} c_{m n}(z)\left[-k_{x}\right] \sin \left[k_{x} x\right] \cos \left[k_{y} y\right] \\
\frac{\partial S}{\partial y}(z) & =\sum_{m=-\infty}^{\infty} \sum_{m=-\infty}^{\infty} c_{m n}(z)\left[-j k_{y}\right] e^{-j\left[k_{x} x+k_{y} y\right]} \\
& =\sum_{m=0}^{\infty} \sum_{m=0}^{\infty} \epsilon_{m n} c_{m n}(z)\left[-k_{y}\right] \cos \left[k_{x} x\right] \sin \left[k_{y} y\right]
\end{aligned}
$$

where $\epsilon_{m n}$ is the double Neumann number:

$$
\epsilon_{m n}= \begin{cases}1, & m=0, n=0 \\ 2, & m=0, n \neq 0 \text { or } m \neq 0, n=0 \\ 4, & m \neq 0, n \neq 0\end{cases}
$$

Note that, by changing the exponential base functions to cosines base functions, the domain of the spectral representation is reduced to $m n[0, \infty]$, thus reducing computational cost four times.

\section{Method Validation and Convergence Analysis}

In [10], authors analyzed a SPCE sensor coupled to a single nanoparticle modeled as a hertzian dipole with current momentum $I_{0} l=1 \mathrm{Am}$. In this analysis, they used the COMSOL Multiphysics, software based on the finite element method, and the DCIM (Discrete Complex Image Method), a semianalytical method used in the spectral representation of the fields in a structure.

In order to verify the validity of the PGF method, we have chosen to test the limiting case in which the nanoparticles are distant from each other in the planar array, comparing the analysis cell with the case studied in [10]. We can use this approach because, in the limiting case, the individual electromagnetic radiation of each cell does not interact with the neighboring cell, converging to the problem of a single 


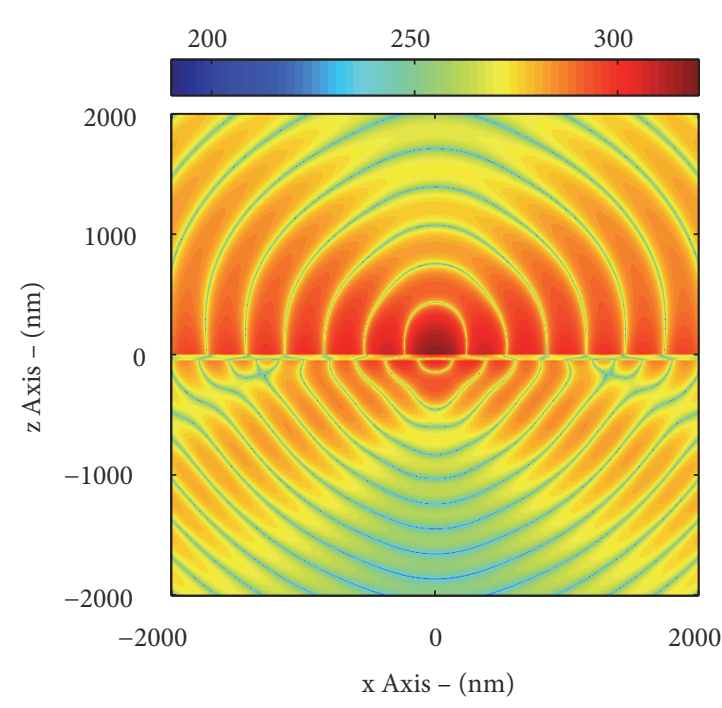

(a)

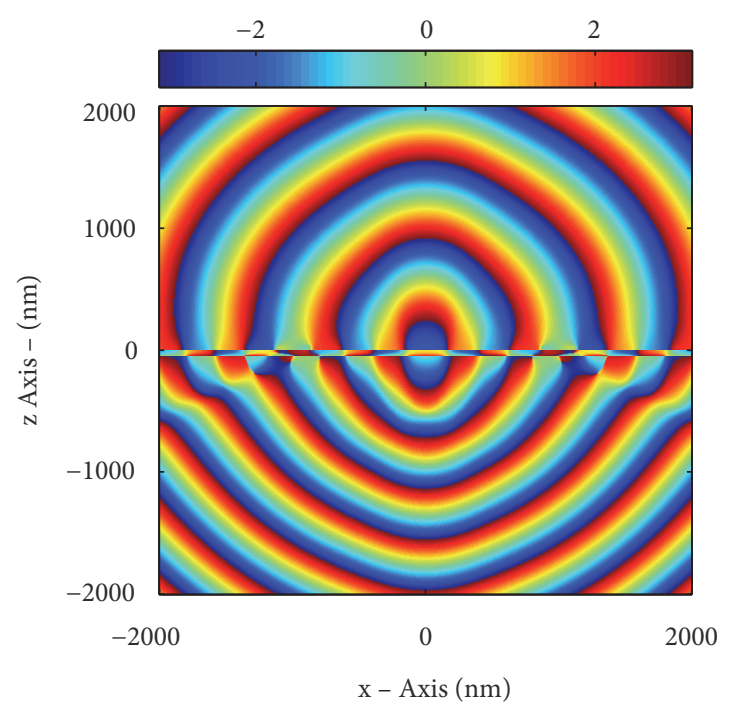

(c)

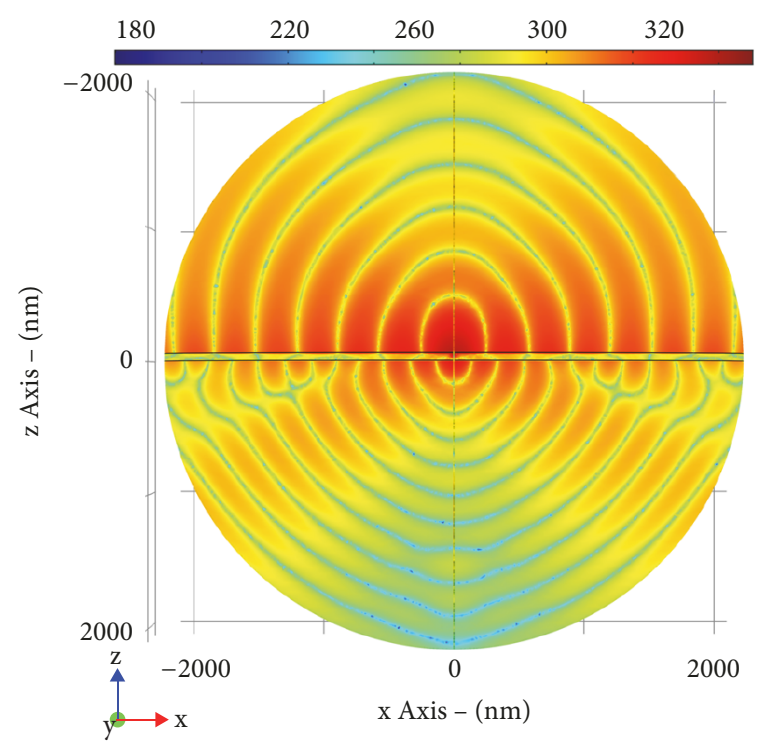

(b)

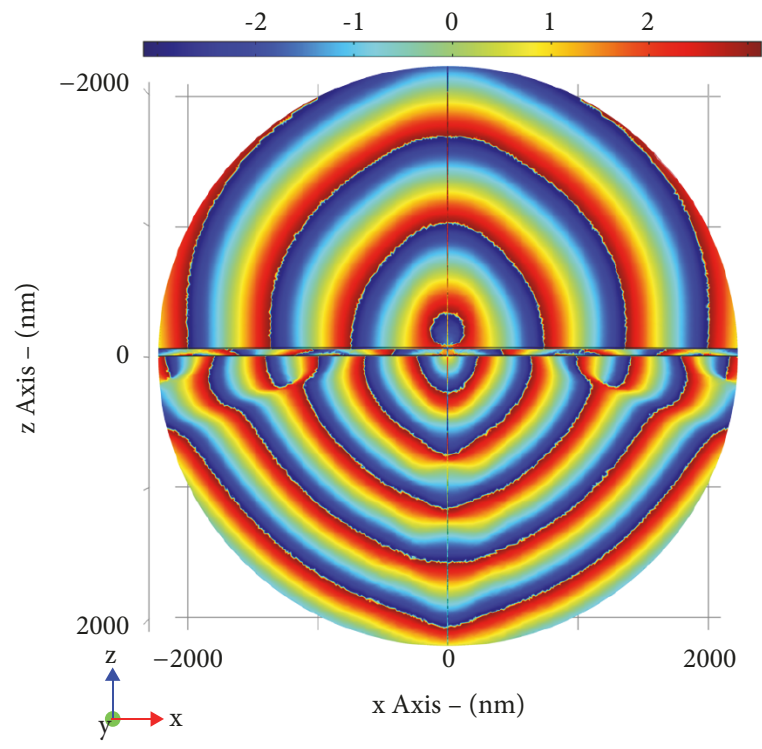

(d)

Figure 5: Component $E_{z}$, Amplitude (dB) $\left(20 \log \left|\operatorname{Re}\left(E_{z}\right)\right|\right)$ : (a) PGF 3D, (b) [10]; Phase (rad) (angle( $\left.\left.E_{z}\right)\right)$ : (c) PGF, (d) [10]. In these results we set the following: $\delta_{g}=50 \mathrm{~nm}, h=20 \mathrm{~nm}, \theta^{\prime}=\phi^{\prime}=0^{\circ}$, and $\lambda=632.8 \mathrm{~nm}$.

nanoparticle excited by an external source. Thus, the CoreShell AuNps were positioned in cells of $\Delta_{c}=60 \mu \mathrm{m}$ and had their current moments normalized by 1 . The amplitude $(\mathrm{dB})$ and phase of $E_{z}$ are shown in Figures 5(a) and 5(c) by PGF method and in Figures 5(b) and 5(d) obtained from COMSOL Multiphysics.

According to [10], simulations performed on a $32 \mathrm{~GB}$ RAM computer with a $3.8 \mathrm{GHz}$ processor required a total time of 5 hours, 12 minutes, and 57 seconds. By the PGF method, the simulation required a time of 17 minutes and 27 seconds. This was done with the discretized mesh at $500 \times 500$, with the series truncated at $m n[0: 150]$ (corresponding to 22801 iterations), on an 8GB RAM computer with a $1.70 \mathrm{GHz}-2.40$ $\mathrm{GHz}$ processor.
We have also compared the results obtained from the numerical integration technique of the spectral representation used in [10] with the PGF method. Figure 15 shows these results for the component $E_{z}$ on the x axis, at $z=y=0$, and on the $\mathrm{z}$ axis, at $x=y=0$.

As can be seen in Figures 5 and 6, the results show good concordance, demonstrating that, in the limit case of low concentration of nanoparticles, the discrete spectrum approaches to the continuous spectrum; consequently, the PGF representation approaches to the representation by the Fourier integral. However, it was necessary to verify the convergence of the method and the necessity of the number of terms in the series of (44). In Figure 7, the convergence of $A_{z}(0,0, z)$ in terms of the number of iterations $m \times n$ 


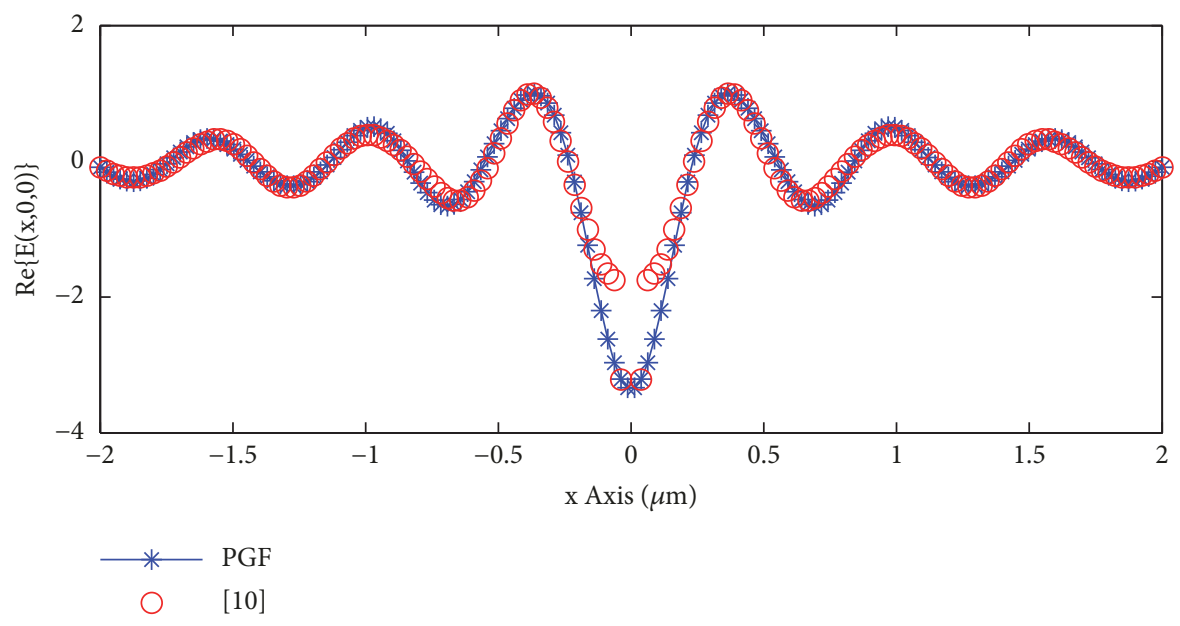

(a)

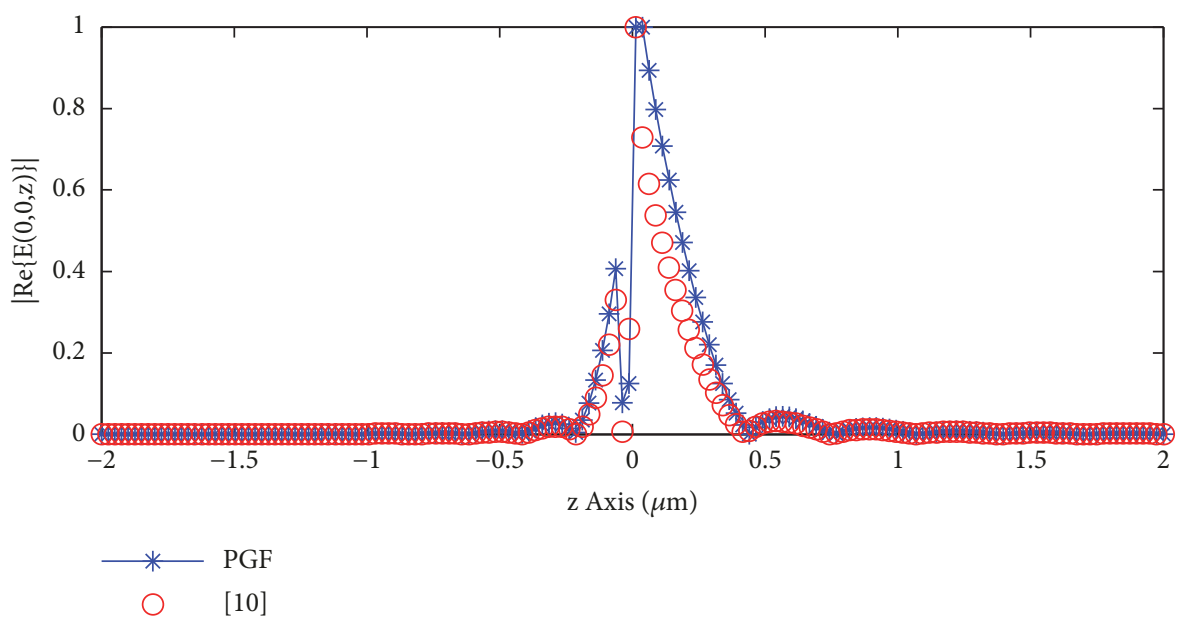

(b)

Figure 6: Results of the PGF and [10] for the component $E_{z}$ : (a) $\operatorname{Re}\left\{E_{z}(x, 0,0)\right\},(\mathrm{b})\left|\operatorname{Re}\left\{E_{z}(0,0, z)\right\}\right|$. In these results we set the following: $\delta_{g}=50 \mathrm{~nm}, h=20 \mathrm{~nm}, \theta^{\prime}=\phi^{\prime}=0^{\circ}$, and $\lambda=632.8 \mathrm{~nm}$.

is demonstrated. To elucidate the convergence in the three media, we choose three points in space: above the gold layer $z=h$, inside the gold layer $z=-\delta_{g} / 2$, and in the prism region $z=\delta_{g}-h$. In the spectral analysis, we will verify that the position of the dominant poles in the spectral representation is function of the cell period. For this reason we will check for three cases of $\Delta_{c}$.

From the graphical results of Figure 7 , we see that the potential field in all situations converges but requires a larger number of terms in the series for the case with larger cell. In fact, as the analysis cell increases, we approach more the limit case, where the discrete spectral representation becomes a continuous spectral representation; in other words, the summation becomes an integral.

\section{Results and Discussion}

Based on the model presented in Section 3, several Matlab codes were developed to simulate the SPCE sensor response. In this section, we considered a reference sample containing only a concentration of Core-Shell AuNps functionalized with fluorophores. Thus, the medium 1 is formed by the periodic planar array of equivalent dipoles, uniformly distributed at a distance $h$ from the surface of the sensor $(z=$ $d_{1}=0$ ) by the chemical spacer. The gold thin film has thickness $\delta_{g}\left(z=d_{2}=-\delta_{g}\right)$. Core-Shell AuNps are excited by an external source of $\lambda=632.8 \mathrm{~nm}$ (suppressed). Thus, the fundamental dipole moment is excited in the direction $\widehat{a}_{r}\left(\theta^{\prime}, \phi^{\prime}\right)$, re-radiating waves on the SPCE sensor structure of the same wavelength. In the periodic array, for simplicity, we consider the cell period in $x$ and $y$ equal, so that $\Delta_{c}=$ $2 a=2 b$ is the period of the cell in the two dimensions. The particularization of the equivalent electromagnetic model is shown in Figure 8.

Considering medium 1, where the nanoparticles are immobilized, as the reference medium and electrically inert (i.e., any variation in the refractive index will be compared with the reference medium), we can approximate their relative permittivity by $\varepsilon_{1}=1$. All the structure is nonmagnetic structure $\left(\mu_{1,2,3}=1\right)$, the permittivity of the prism (BK7 optical glass) is $\varepsilon_{3}=2.30$, and the gold layer is $\varepsilon_{2}=-11.63-$ $1.34 j$, with the latter described by the Lorentz-Drude model 


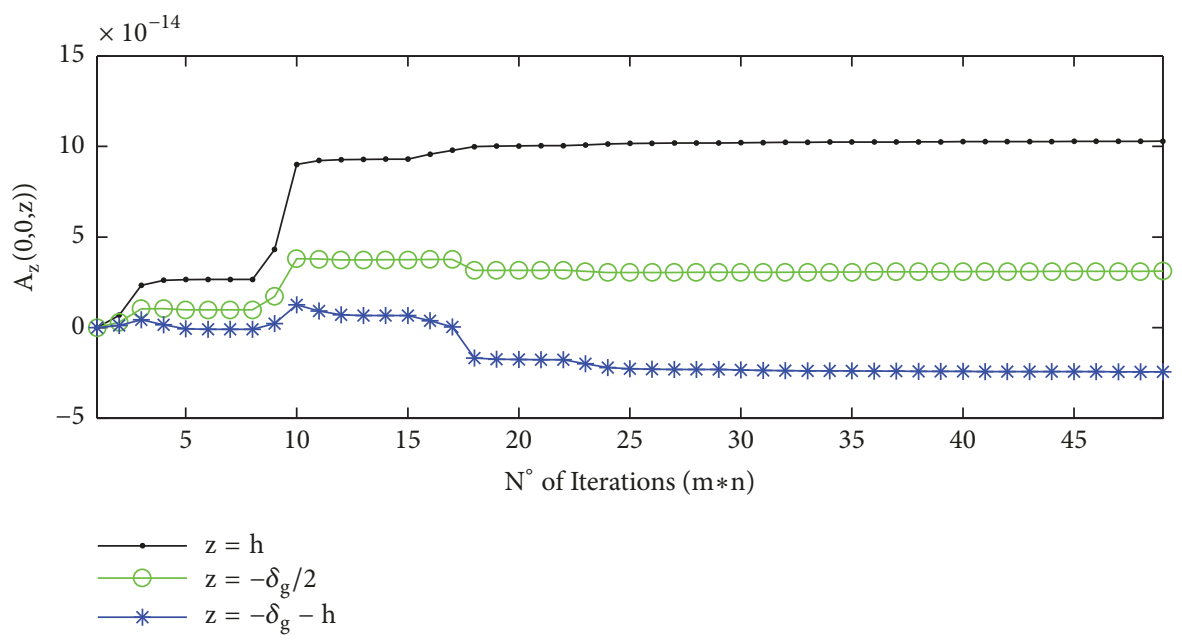

(a)
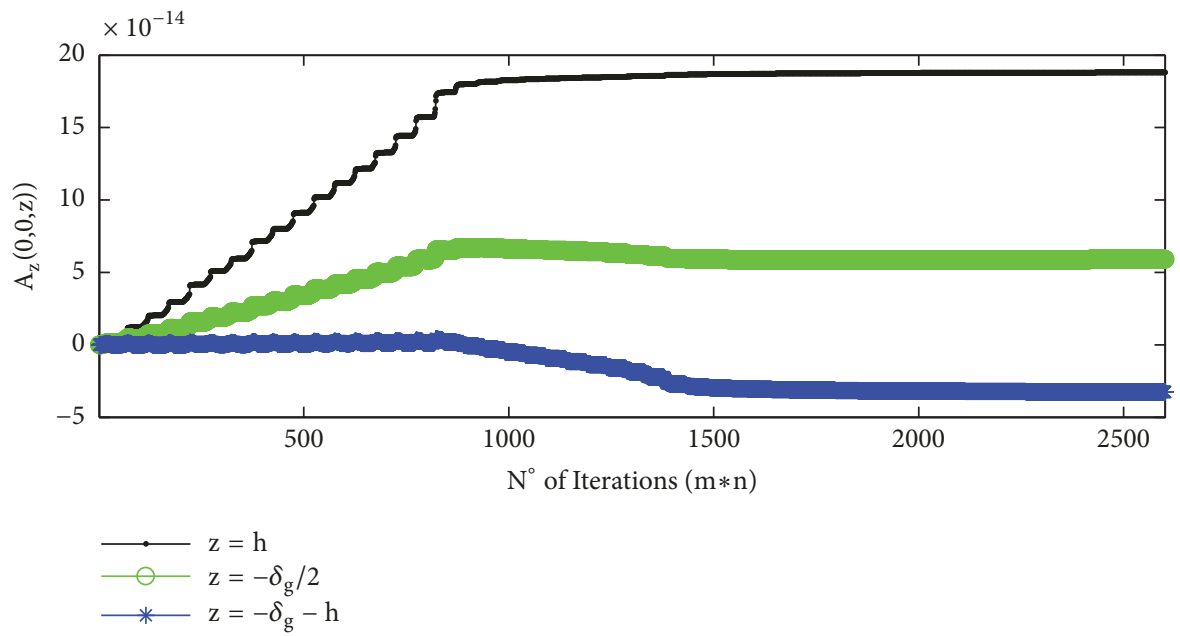

(b)
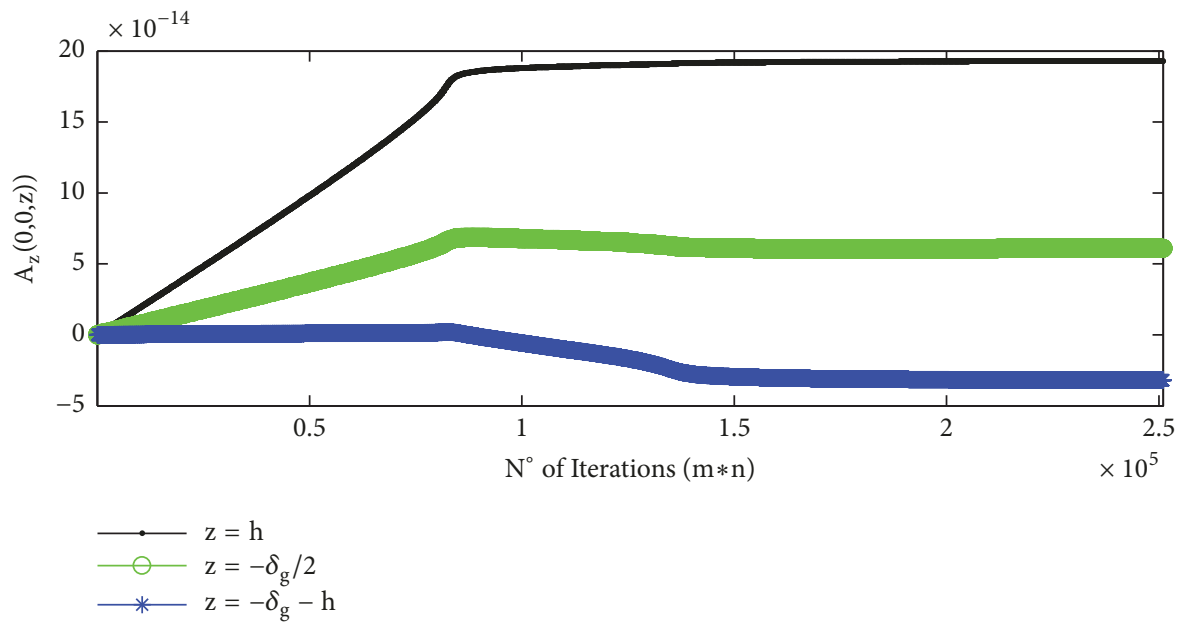

(c)

FigURE 7: $A_{z}(0,0, z)$ convergence for the following: (a) $\Delta_{c}=1 \mu m,(\mathrm{~b}) \Delta_{c}=10 \mu \mathrm{m}$, and (c) $\Delta_{c}=100 \mu m$. In these results we set the following: $\delta_{g}=50 \mathrm{~nm}, h=20 \mathrm{~nm}, \theta^{\prime}=\phi^{\prime}=0^{\circ}$, and $\lambda=632.8 \mathrm{~nm}$. 


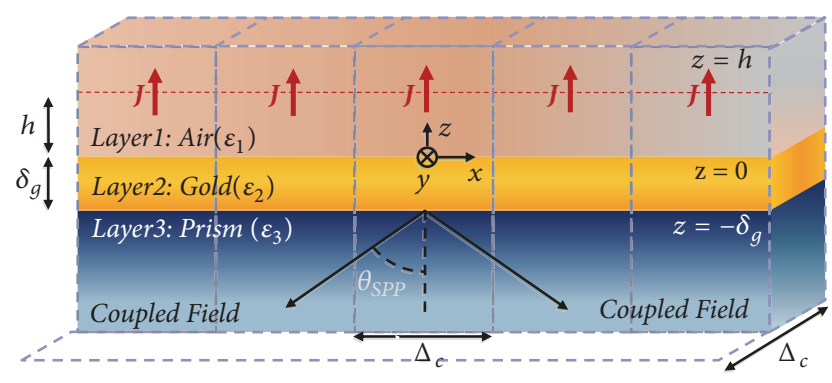

Figure 8: Particularization of the equivalent electromagnetic model for the SPCE sensor.

with excellent accuracy. In this step, we do not intend to verify the influence of the AuNPs dimensions on the optical response of the sensor, but we must keep in mind the near field amplification already verified that these nanoparticles suffer by the LSPR phenomenon. As an effective dipole moment, we find $p_{\text {eff }}=(7.17+j 0.003)$ Debye, approximated by (1) and (2), for a particle with gold core and dielectric shell $r_{c}=20 \mathrm{~nm}, \delta_{c}=10 \mathrm{~nm}$, respectively, aggregated with a fluorophore with polarizability $\alpha_{F F}=(7.1 \pm 1.1)$ Debye $/ \mathrm{Vm}^{-1}$ [19].

5.1. Spatial Analysis of Magnetic Potential and Electromagnetic Fields. First, the magnetic potential field (16) was used to verify the influence of the gold layer thickness to $\delta_{g}=$ $10 \mathrm{~nm}, 50 \mathrm{~nm}$, and $100 \mathrm{~nm}$, with the dipole at a height $h=$ $200 \mathrm{~nm}$, oriented in three distinct directions, $\theta=0^{\circ}, 45^{\circ}$, and $90^{\circ}$, with $\phi^{\prime}=0^{\circ}$, in the analysis cell with period $\Delta_{c}=$ $10 \mu \mathrm{m}$. The graphs were generated in the $x z$ plane at $y=$ 0 , for the normal component $A_{z}$ of the magnetic potential field.

For better visualization and analysis, the graphical results in Figure 9 were normalized to $[-2,6] \times 10^{-14} \mathrm{~Wb} / \mathrm{m}$ and limited to $[-2,2] \mu m$ in $x z$ plane. The main objective of these analyses is to verify the influence of the thickness of the gold layer $\delta_{g}$ and the orientation of the equivalent dipoles defined by the angles $\theta^{\prime}$ and $\phi^{\prime}$. We can verify that, as the orientation of the dipoles changes from $\theta^{\prime}=0^{\circ}$ to $\theta^{\prime}=90^{\circ}$, less the normal component $A_{z}$ contributes to the magnetic potential field. This is in accordance with (18)-(26), where we can show that, in general $\left|\widetilde{R}^{T M}\right|>\left|S^{E M}\right|$, in other words, the component $A_{z}$ is more effectively excited by $J_{z}$ than $J_{x}$ or $J_{y}$. In general, for all cases with different dipole orientation, we have a better field transmission in the region of the dielectric prism for $\delta_{g}=10 \mathrm{~nm}$. In the case where $\delta_{g}=100 \mathrm{~nm}$, we have a larger field reflection in the sample region, which may reduce the signal reading at the SPCE sensor output. However, for this gold thickness, we can verify the appearance of SPP waves, mainly for vertical orientation. We found the best thickness of $\delta_{g}=50 \mathrm{~nm}$, where we have the two basic needs in the sensor structure at the same time: the considerable capacity of transmitting TM radiation waves through the prisma medium and the excitation of SPP waves on the gold layer.

From the differential relations (38) and (39), we can now analyze the Electric and Magnetic fields in Figure 10.
In order to verify the influence of the dipole orientation in the array, electric and magnetic field results of the $E_{x}, E_{z}$, and $H_{y}$ components were generated for the orientations $\theta^{\prime}=$ $0^{\circ}$ (VED: Vertical Electric Dipole), intermediate $\theta^{\prime}=45^{\circ}$, and $\theta^{\prime}=90^{\circ}$ (HED: Horizontal Electric Dipole), with the array positioned at a height $h=20 \mathrm{~nm}$ in a cell of period $\Delta_{c}=10 \mu m$ (Figure 10). For better visualization, the fields were normalized to $[-50,50] d B$ and limited to $[-2,2] \mu m x$ and $z$.

Differently from the results of magnetic potential (Figure 9), we can now verify more accurately the inclination of the equivalent dipole for the VED, intermediate, and HED cases. For the HED case (Figures 10(c), 10(f), and 10(i)), we can see a considerable field transmission in the prism region. However, note that there is no excitation of SPP waves at the sample/gold interface; that is, the field comes only from the dipole radiation, and it has no directional characteristic as a function of SPP wave excitation. For the intermediate case (Figures 10(b), 10(e), and 10(h)), the considerable excitation of SPP waves and asymmetric field characteristic in the three media is verified. In the VED orientation (Figures 10(a), $10(\mathrm{~d})$, and $10(\mathrm{~g})$ ), also a considerable SPP wave component in the sample-gold interface is verified. This is due to the light excitation of TM waves in the VED case, in accordance with (18)-(26) and the TM nature of the SPP waves. The radiation in the prism region has a highly directional characteristic, being derived from the radiation of the VED coupled by the excitation of the SPP waves.

In summary, we can see that the HED mode, which predominantly excites TE modes, does not efficiently excite plasmons. In contrast, the VED mode actively excites SPP waves, thus, coupling highly polarized TM waves in the prism region, with the latter being the mode that presents the best feature in relation to the SPCE sensor. From this point, we will adopt the VED polarization as effective and consider only the case $\theta^{\prime}=0$. This choice cancels the tangential components of the magnetic potential field $\left(A_{x}\right.$ and $\left.A_{y}\right)$, leaving only the normal component $A_{z}$ :

$$
\mathbf{A}_{1,2,3}(\mathbf{r})=\sum_{m=0}^{\infty} \sum_{n=0}^{\infty} \epsilon_{m n} A_{z z_{1,2,3}}^{m n}(z) \cos \left[k_{x} x\right] \cos \left[k_{y} y\right]
$$

Finally, it is worth showing that the periodic conditions are met, for both the electric and magnetic field. Figure 11 shows the field result for two cells with period $\Delta_{c}=4 \mu \mathrm{m}$, in the $x y$ plane at $z=0$ and $x z$ plane at $y=0$.

From this result, the interaction (interference) between the fields re-irradiated by two near dipoles is verified, where, at the boundary of the cells of analysis, the periodic boundary conditions are obeyed.

5.2. Spectral Analysis. Originally, in the inverse double transform of the Fourier series, which defines the magnetic potential at (45), the sum of complex exponentials with the double summation is performed by $m=-\infty:+\infty$ and $n=$ $-\infty$ : $+\infty$, defining the discrete spectral domain $m n$. It was proposed to use Euler's identity to change the representation in terms of exponentials by a representation in terms of cosine functions, thus reducing the spectral domain to $m=0:+\infty$ 


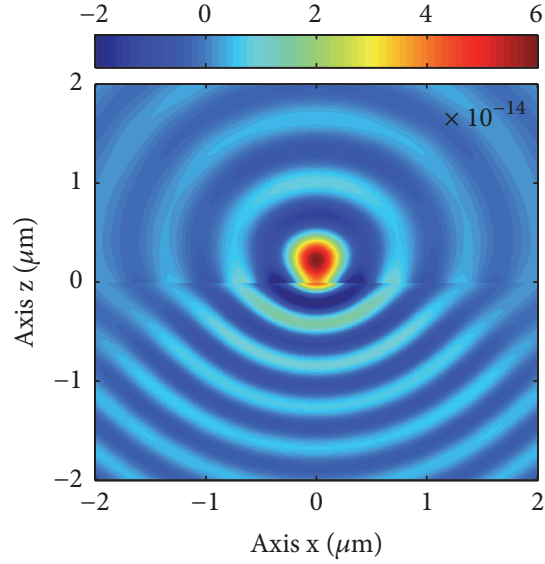

(a)

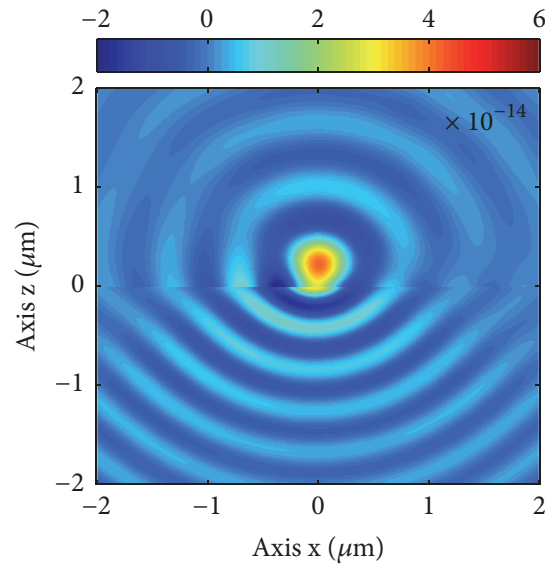

(d)

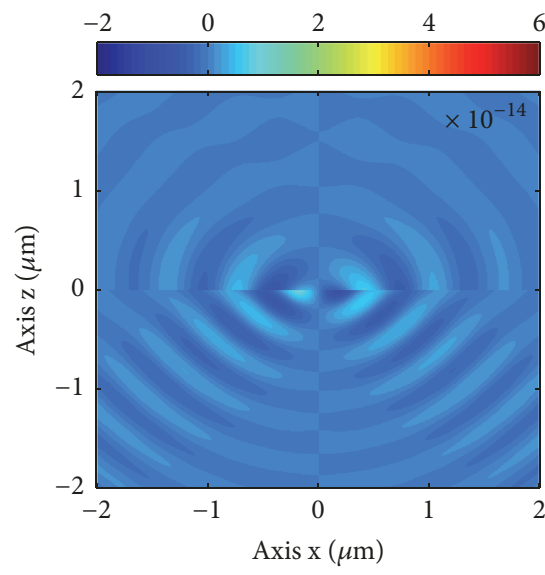

(g)

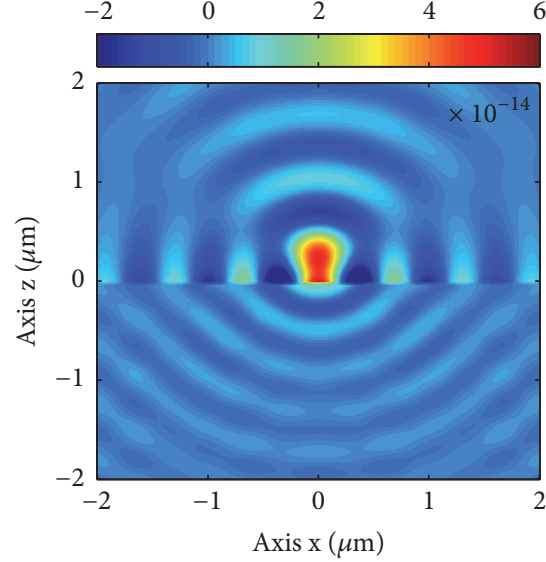

(b)

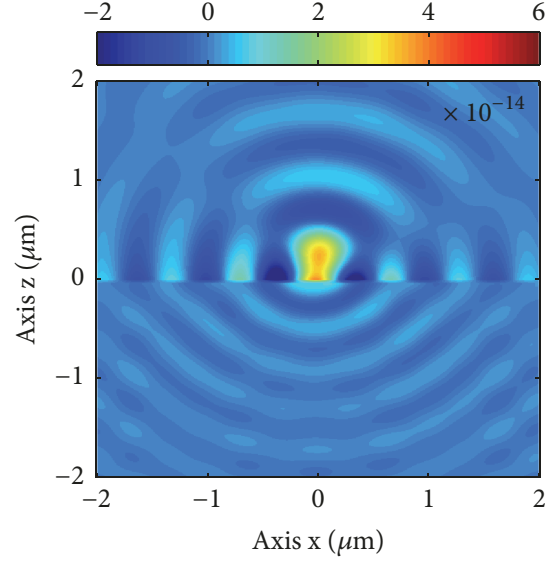

(e)

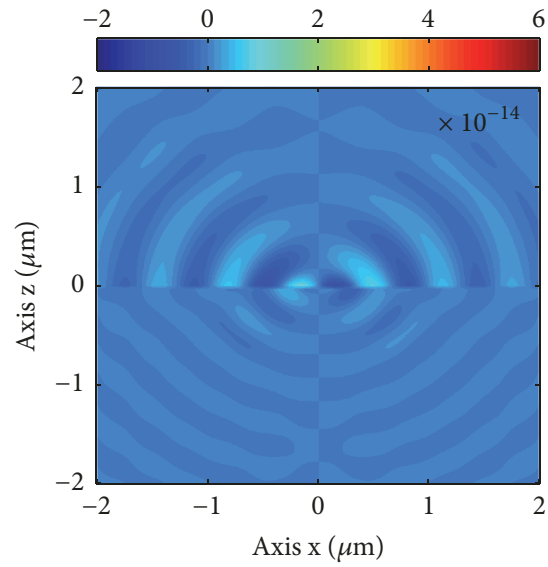

(h)

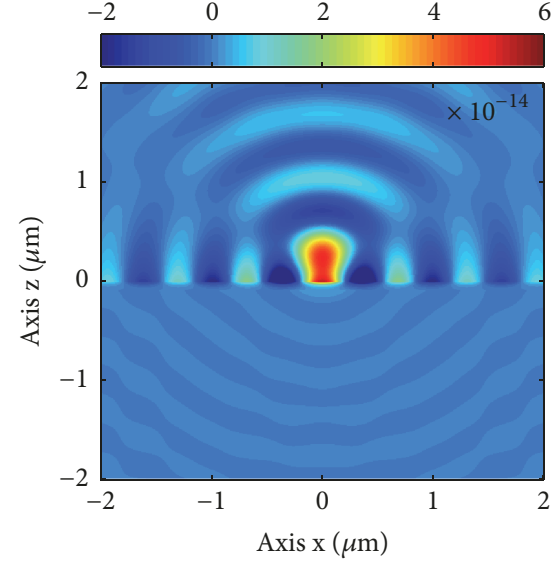

(c)

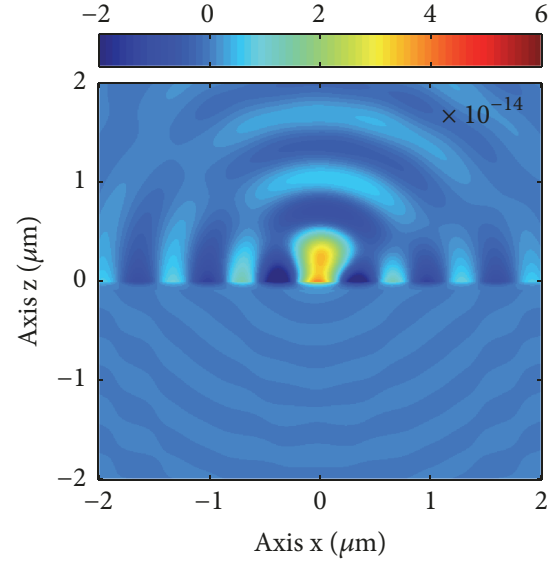

(f)

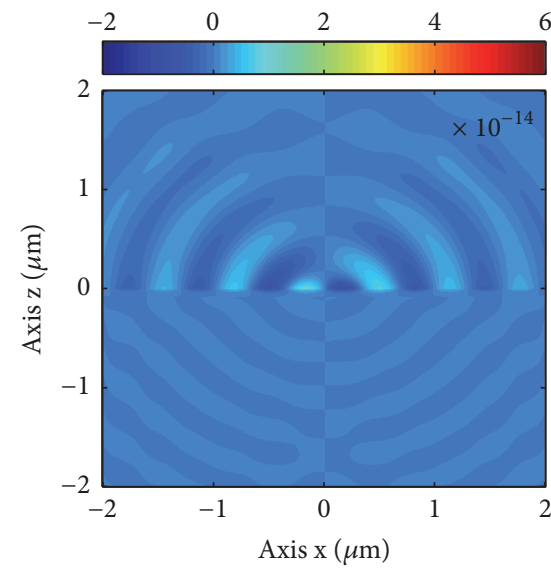

(i)

Figure 9: Magnetic Potential Field $A_{z}$ for $\theta^{\prime}=0^{\circ}:$ (a) $\delta_{g}=10 \mathrm{~nm}$, (b) $\delta_{g}=50 \mathrm{~nm}$, and (c) $\delta_{g}=100 \mathrm{~nm} ; \theta^{\prime}=45^{\circ}:(\mathrm{d}) \delta_{g}=10 \mathrm{~nm}$, (e) $\delta_{g}=50 \mathrm{~nm}$, and (f) $\delta_{g}=100 \mathrm{~nm} ; \theta^{\prime}=90^{\circ}:(\mathrm{g}) \delta_{g}=10 \mathrm{~nm}$, (h) $\delta_{g}=50 \mathrm{~nm}$, and (i) $\delta_{g}=100 \mathrm{~nm}$. In these results we set the following: $h=200 \mathrm{~nm}, \Delta_{c}=10 \mu \mathrm{m}, \lambda=632.8 \mathrm{~nm}$, and $\phi^{\prime}=0^{\circ}$.

and $n=0:+\infty$, reducing the computational cost. In other words, the coefficients of (45) are even functions of $m$ and $n$.

The analysis of the spectral terms $A_{z z}^{m n}$ and the generalized reflection and transmission coefficients can give us important information about the dominant terms in the representation of the potential field (45). In Figure 12 the spectral distributions of the generalized reflection and transmission coefficients in the $m n$ plane are shown for $\Delta_{c}=1 \mu m, \Delta_{c}=$ $10 \mu \mathrm{m}$, and $\Delta_{c}=100 \mu \mathrm{m}$. 


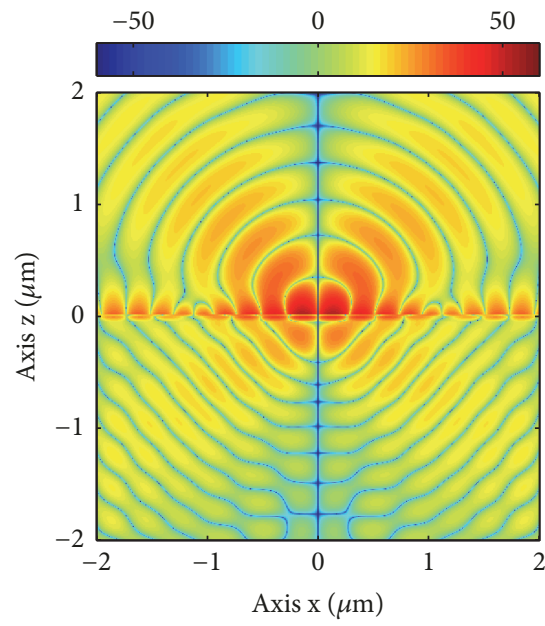

(a)

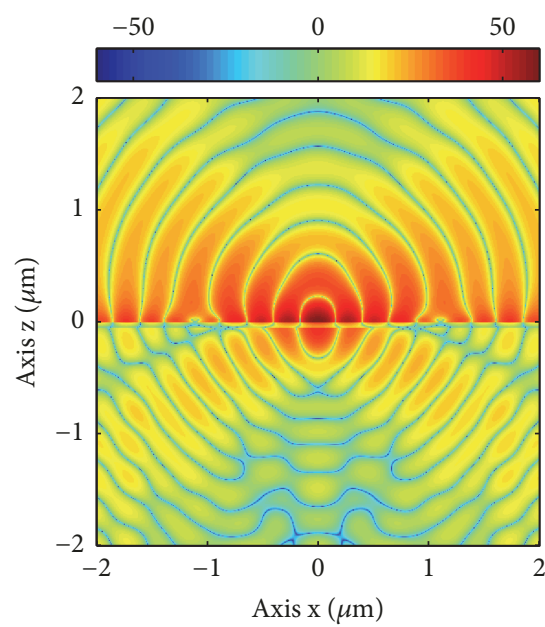

(d)

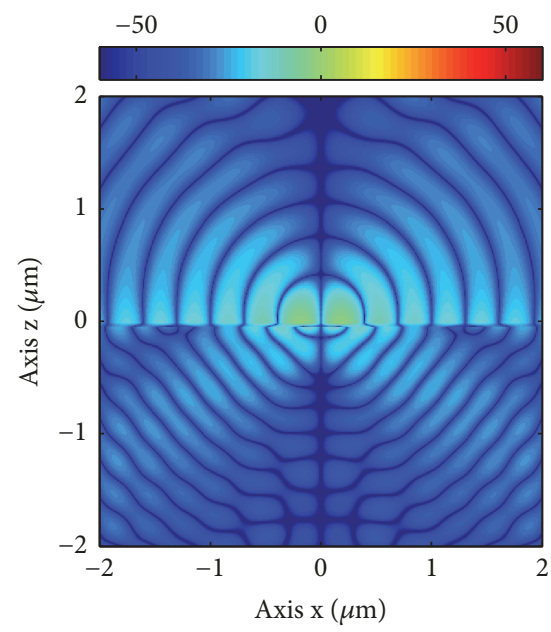

(g)

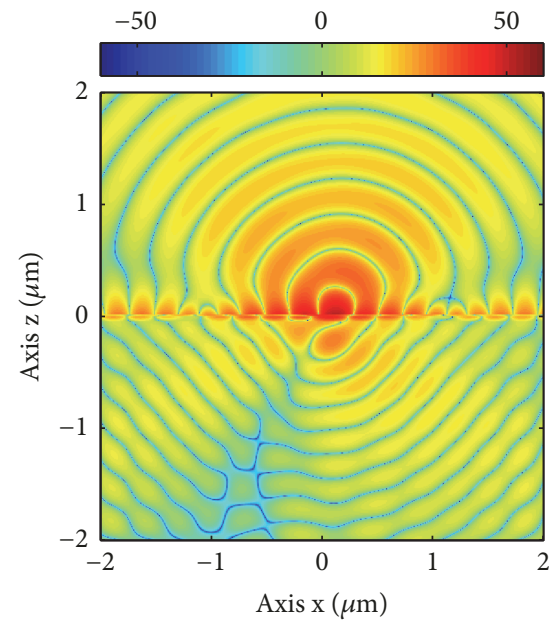

(b)

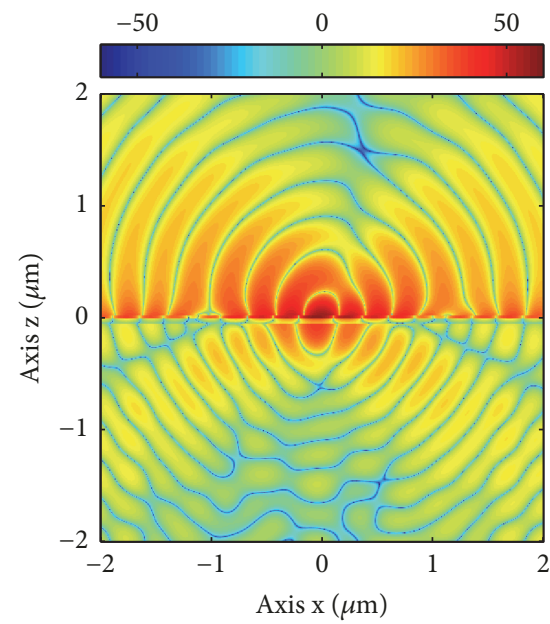

(e)

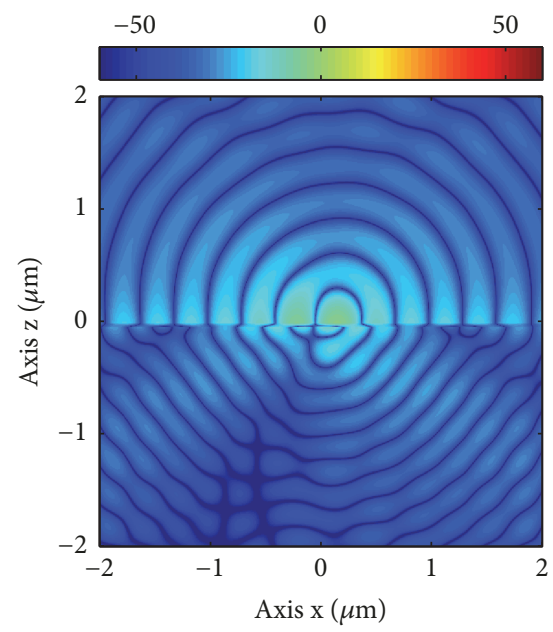

(h)

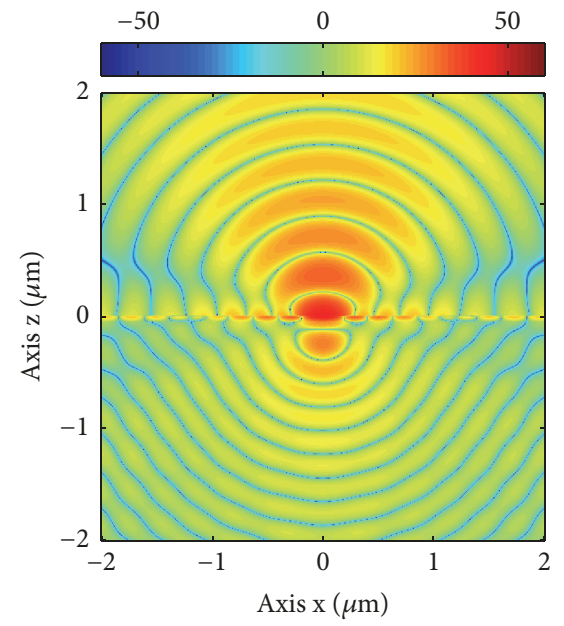

(c)

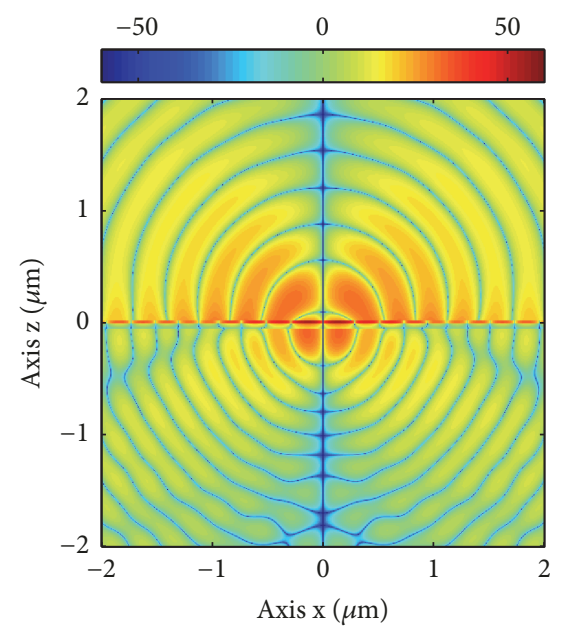

(f)

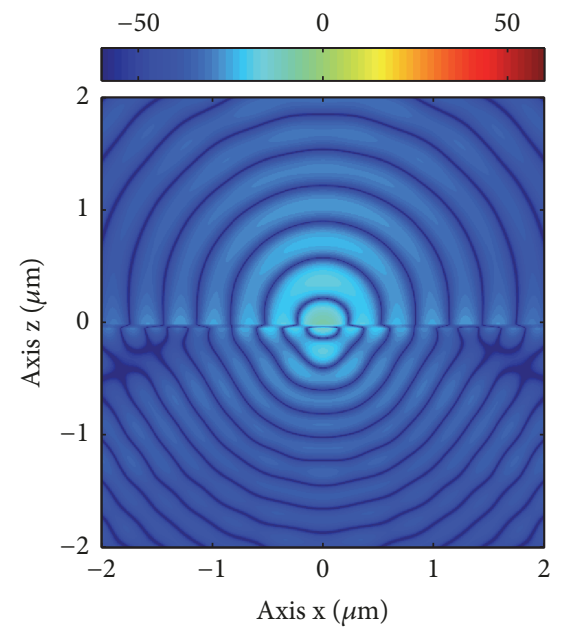

(i)

Figure 10: Electric and Magnetic Field. $20 \log |\operatorname{Re}\{E x\}|:$ (a) $\theta^{\prime}=0^{\circ}$, (b) $\theta^{\prime}=45^{\circ}$, and (c) $\theta^{\prime}=90^{\circ} ; 20 \log |\operatorname{Re}\{E z\}|:\left(\right.$ d) $\theta^{\prime}=0^{\circ},(\mathrm{e}) \theta^{\prime}=45^{\circ}$, and (f) $\theta^{\prime}=90^{\circ} ; 20 \log |\operatorname{Re}\{H y\}|:(\mathrm{g}) \theta^{\prime}=0^{\circ}$, (h) $\theta^{\prime}=45^{\circ}$, and (i) $\theta^{\prime}=90^{\circ}$. In these results we set the following: $\delta_{g}=50 \mathrm{~nm}, h=20 \mathrm{~nm}$, $\Delta_{c}=10 \mu m, \lambda=632.8 \mathrm{~nm}$, and $\phi^{\prime}=0^{\circ}$. 


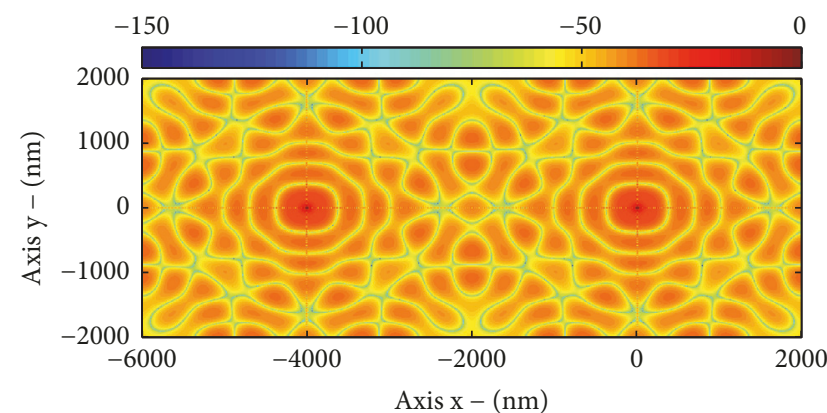

(a)

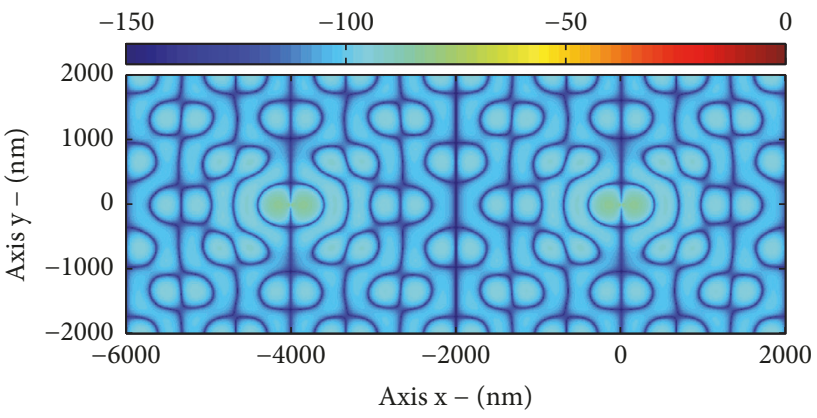

(c)

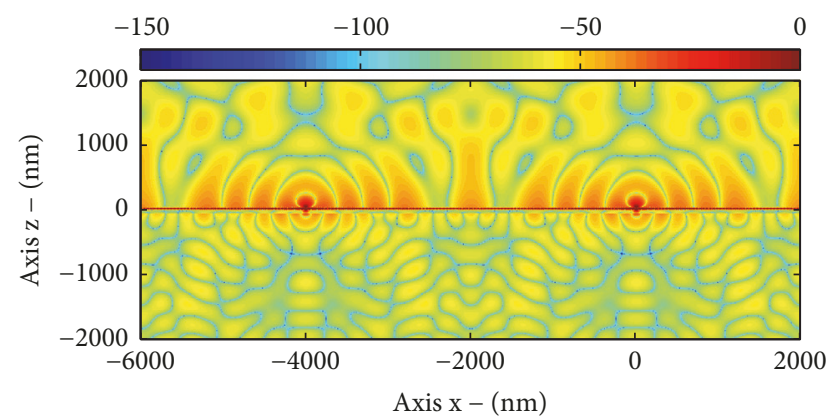

(b)

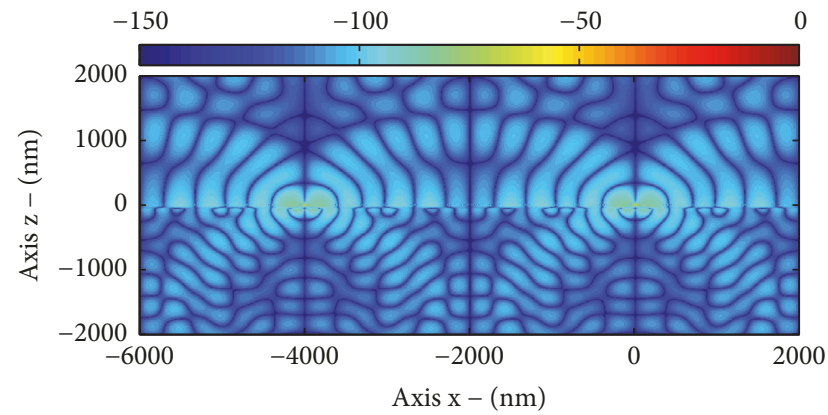

(d)

Figure 11: Component $z$ of the electric field $20 \log |\operatorname{Re}\{E z\}|$, in $\mathrm{dB}$, in the planes: (a) $x y$ and (b) $x z$; Component $y$ of the magnetic field $20 \log |\operatorname{Re}\{H y\}|$, in $\mathrm{dB}$, in the planes: (c) $x y$ and (d) $x z$. In these results we set the following: $\delta_{g}=50 \mathrm{~nm}, h=20 \mathrm{~nm}, \Delta_{c}=4 \mu \mathrm{m}, \lambda=632.8 \mathrm{~nm}$, $\theta^{\prime}=0^{\circ}$, and $\phi^{\prime}=0^{\circ}$.

For the case of Figures 12(a) and 12(d), the terms of greater dominance are close to the origin and converge rapidly to fixed values. Also, in these cases, the spectrum does not present a cylindrical symmetry with $\mathrm{mn}$ because the geometry of the AuNps array is rectangular. In the case of Figures 12(c) and 12(f), we can verify that the dominance terms (peaks of resonance) occur far from the origin. Also, we observe in these cases a cylindrical symmetry in the spectrum $m n$. This happens because, for larger $\Delta_{c}$, the AuNps array converges (behaves) to an isolated single dipole, which presents cylindrical symmetry in the $x y$ plane, and consequently, a cylindrical symmetry in the $m n$ plane. This characteristic of cylindrical convergence of the spectrum will be considered in the next paragraphs.

A fundamental result is the characteristic of cylindrical symmetry that the spectral terms present. This is because the eigenvalues $k_{x}$ and $k_{y}$ have the same structure, and they perform similar roles in the spectral representation. Due to symmetry, we can express the eigenvalues by a $k_{\rho}=\sqrt{k_{x}^{2}+k_{y}^{2}}$ and express the propagation constant $k_{z_{u}}=\sqrt{k_{u}^{2}-k_{\rho}^{2}}$. Also, due to the cylindrical symmetry, we can limit the spectral analysis to the domain $m n[0,+\infty]$. Even stronger, we can fix $n$ at any value and perform the one-dimensional analysis in $\mathrm{m}$. For $n=0$, we obtain $k_{\rho}=\left[2 \pi m / \Delta_{c}\right]$.

Setting $n=0$, for $\Delta_{c}=1 \mu \mathrm{m}, 10 \mu \mathrm{m}$ and $100 \mu \mathrm{m}$, Figure 13 shows the generalized reflection and transmission coefficients and in Figure 14 the spectral terms of the potential field component $A_{z}$, all in the discrete spectral domain $m$, in the range $[0, M]$.

Figure 13 shows the rise of two resonance peaks; these points arise from the poles of the generalized coefficients at the interfaces of the structure. Therefore, the spectral position of these resonances is dependent on the thickness of the gold layer. However, [10] found that for $\delta_{g}=5 \mathrm{~nm}$ the poles of the generalized and Fresnel coefficients are practically the same. Thus, the first resonance comes from the $R_{21}^{T M}\left(\varepsilon_{2} k_{z 1}+\right.$ $\left.\varepsilon_{1} k_{z 2}=0\right)$ and is related to the surface plasmon mode at the Air/Gold interface. The second resonance is the result of the $R_{23}^{T M}\left(\varepsilon_{2} k_{z 3}+\varepsilon_{3} k_{z 2}=0\right)$, being related to the surface plasmon wave mode that appears in the Gold/Prism interface. These are the two dominant modes in the spectral representation of the fields in (44). An approximate way to determine the spectral position of these poles in $m$ is from the dispersion relation at the interfaces, given that any $n$. Thus, the poles $m_{S P P 1}$ and $m_{S P P 2}$ are located, respectively, in (46) and (47).

$$
\begin{aligned}
& m_{S P P 1}=\operatorname{Re}\left\{\frac{\Delta_{c} k_{0}}{2 \pi} \sqrt{\frac{\varepsilon_{r 2} \varepsilon_{r 1}}{\varepsilon_{r 2}+\varepsilon_{r 1}}-\left[\frac{2 \pi n}{\Delta_{c} k_{0}}\right]^{2}}\right\} \\
& m_{S P P 2}=\operatorname{Re}\left\{\frac{\Delta_{c} k_{0}}{2 \pi} \sqrt{\frac{\varepsilon_{r 3} \varepsilon_{r 2}}{\varepsilon_{r 3}+\varepsilon_{r 2}}-\left[\frac{2 \pi n}{\Delta_{c} k_{0}}\right]^{2}}\right\}
\end{aligned}
$$

where $k_{0}$ is the propagation constant of the free space and $\varepsilon_{r u}$ the relative permissiveness of the medium $u(u=1,2,3)$. From Figure 14, we can verify that the pole $m_{S P P 1}$ is more 


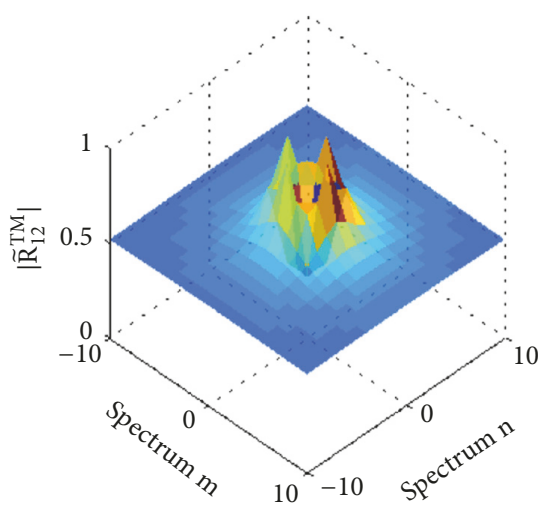

(a)

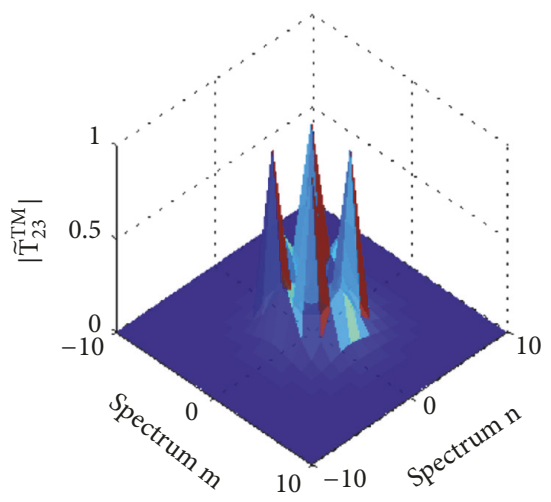

(d)

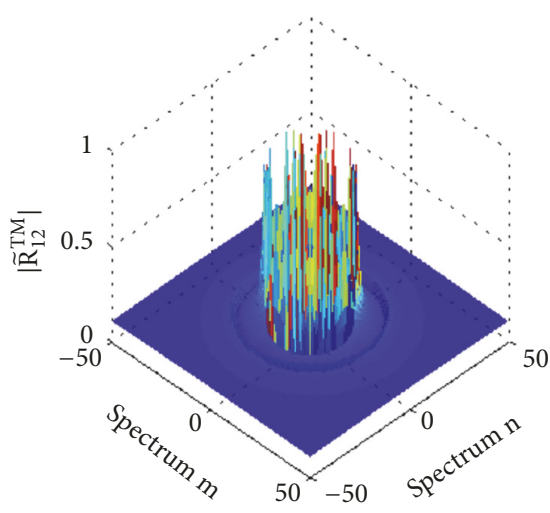

(b)

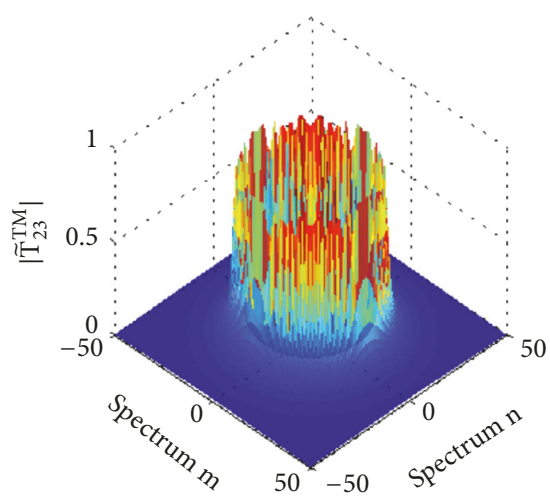

(e)

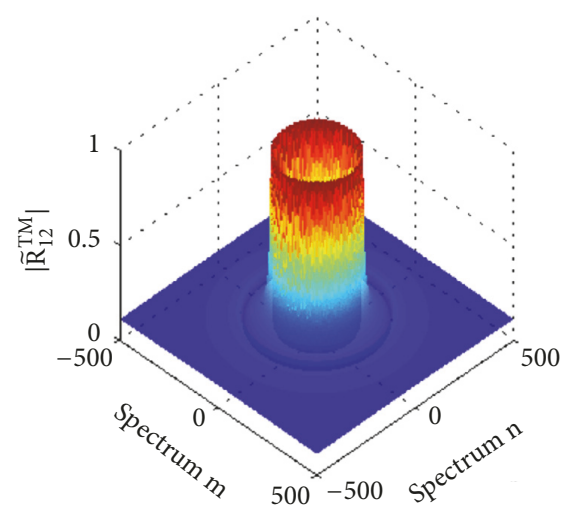

(c)

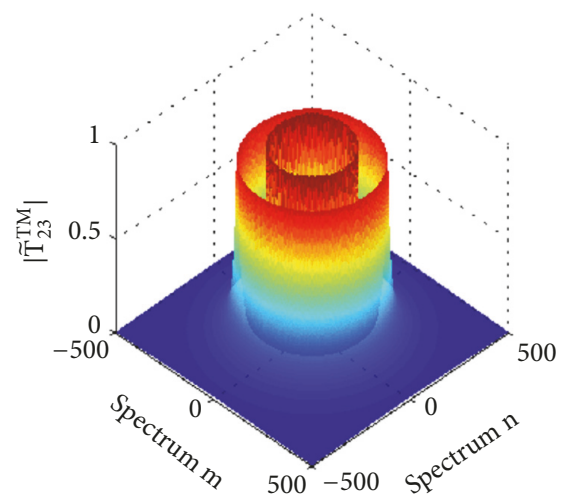

(f)

FIGURE 12: Spectral distribution in the mn plane: $\left|\widetilde{R}_{12}^{T M}\right|$ for (a) $\Delta_{c}=1 \mu m$, (b) $\Delta_{c}=10 \mu m$, and (c) $\Delta_{c}=100 \mu m$; and $\left|\widetilde{T}_{23}^{T M}\right|$ for (d) $\Delta_{c}=1 \mu m$, (e) $\Delta_{c}=10 \mu \mathrm{m}$, and (f) $\Delta_{c}=100 \mu \mathrm{m}$. In these results we set the following: $\delta_{g}=50 \mathrm{~nm}$.

intense than $m_{S P P 2}$; for this reason, this will be the dominant pole in the calculation of the plasmon propagation constant $k_{\rho}^{S P P}$. In fact, since $k_{\rho}^{S P P}=\left(2 \pi m_{S P P 1}\right) / \Delta_{c}$, we can approximate the resonance plasmonic angle by

$$
\theta_{S P P} \simeq \sin ^{-1}\left[\frac{k_{\rho}^{S P P}}{k_{3}}\right]
$$

It is also seen that, as the period of the cell $\Delta_{c}$ increases, there is a shift to the right of the resonances, which, in terms of the spectral representation, means a greater amount of terms to be computed in the sum of (45).

The results in Figure 14 were generated for the spectral terms of the magnetic potential at three points in the $\mathrm{z}$-space, above the gold layer $z=h$, inside the gold layer $z=-\delta_{g} / 2$, and below the gold layer $z=-\left(\delta_{g}+h\right)$. For $\Delta_{c}=1 \mu m$, at $n=0$, the SPP poles appear in $m_{S P P 1}=2$ and $m_{S P P 2}=$ 3 , the plasmon propagation constant is $k_{\rho}^{S P P}=1.2660 k_{0}$, and the plasmonic coupling angle $\theta_{S P P}=56.57^{\circ}$. Note that convergence occurs rapidly in $m$ approximately $M=10$. For $\Delta_{c}=10 \mu m$, the SPP poles appear in $m_{S P P 1}=17$ and $m_{S P P 2}=$ 27 , the plasmon propagation constant is $k_{\rho}^{S P P}=1.0761 k_{0}$, and the plasmonic coupling angle $\theta_{S P P}=45.18^{\circ}$. For $\Delta_{c}=100 \mu \mathrm{m}$, the SPP poles appear in $m_{S P P 1}=165$ and $m_{S P P 2}=267$, the plasmon propagation constant is $k_{\rho}^{S P P}=1.0444 k_{0}$, and the plasmonic coupling angle $\theta_{S P P}=43.51^{\circ}$.

As the cell period increases, we approach the limiting case, where the radiation comes from a single isolated particle. This case was studied in [10], where a plasmon propagation constant $k_{\rho}^{S P P}=(1.0458-j 0.0051) k_{0}$ and plasmonic coupling angle $43.7^{\circ}$ were obtained. Note that the results found for $\Delta_{c}=100 \mu \mathrm{m}$ are close to the results obtained in the limit case in [10]. It is also verified that the discrete spectrum gains characteristic of the continuous spectrum as we approach the limiting case.

In order to verify the effect of the SPP1 and SPP2 poles for different values of term $m$, in the spatial domain, Figure 15 shows the electric and magnetic field graphs to $\Delta_{c}=10 \mu \mathrm{m}$, for terms $m=7, m=m_{S P P 1}=17$ and $m=m_{S P P 2}=27$, with $n=0$.

By the graphical analysis of the spectral distribution in Figures 13 and 14, we can note that for points near the sensor the spectral terms $m>m_{S P P 2}$ contributes less to the composition of the total field. This information gives us a good idea of the number of terms needed for the field representation in a cell. In Figure 16 the electric and magnetic fields are shown for $\Delta_{c}=10 \mu \mathrm{m}$, with the truncated series $m=0: 50$ and $n=0: 50$, which corresponds to $51^{2}$ terms in the spectral representation. 


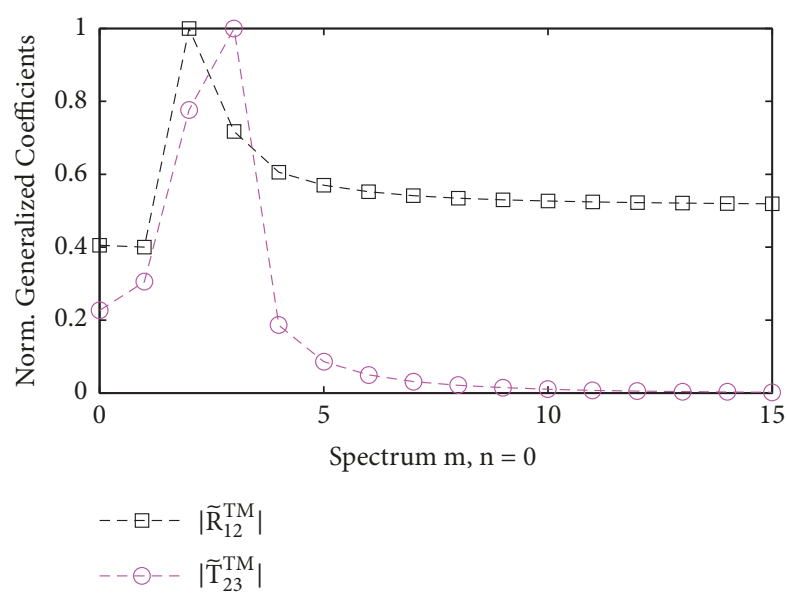

(a)

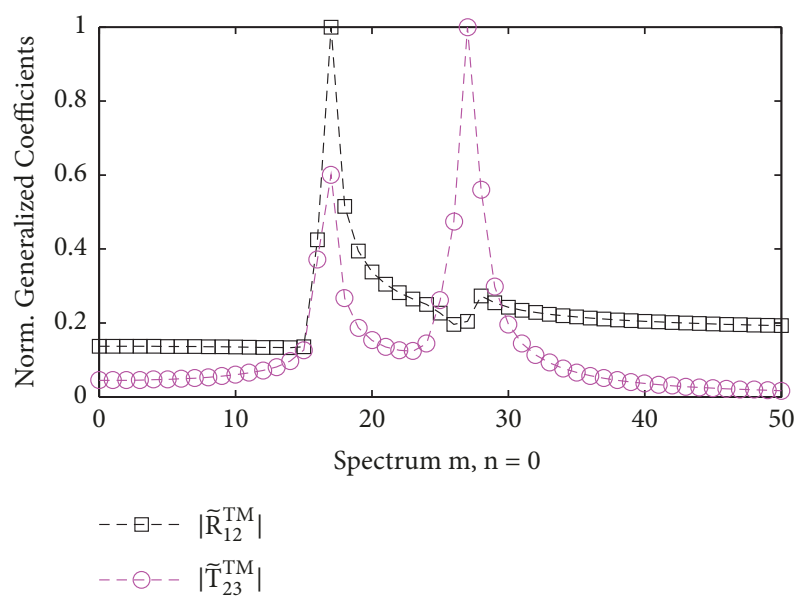

(b)

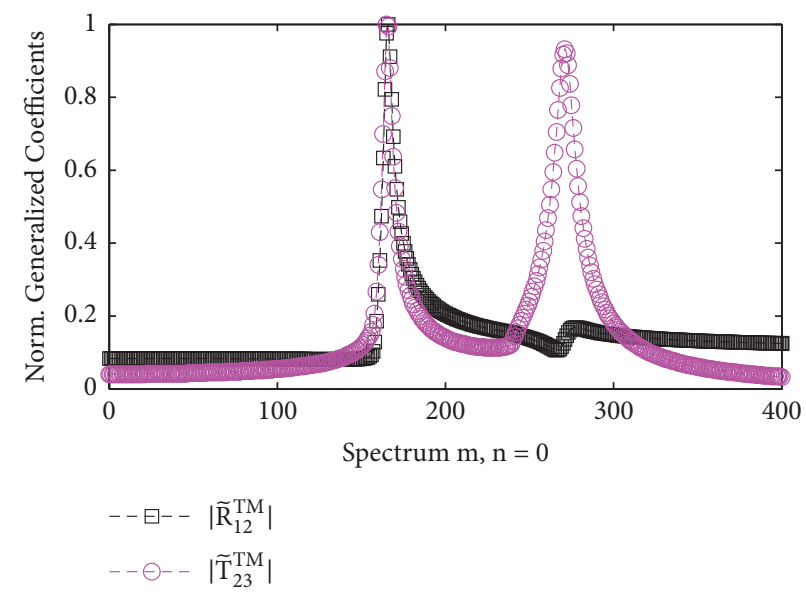

(c)

FIGURE 13: Normalized spectral distribution of $\left|\widetilde{R}_{21}^{T M}\right|$ and $\left|\widetilde{T}_{23}^{T M}\right|$ in $m$, with $n=0$, for (a) $\Delta_{c}=1 \mu m$; (b) $\Delta_{c}=10 \mu m$; (c) $\Delta_{c}=100 \mu m$. In these results we set the following: $\delta_{g}=50 \mathrm{~nm}$ and $\lambda=632.8 \mathrm{~nm}$.

For better visualization the graphs in Figure 15 were generated in the $x z$ plane $[-2,2] \mu \mathrm{m}$. For the three cases of $\Delta_{c}$, we can verify that the three types of fields contribute in different ways to the total field in the sensor structure. The SPP1 pole contributes to field coupling in the prism region, a fundamental feature of the SPCE sensor. Note that the field distribution in these cases is equivalent to SPP waves excited by plane waves at metal/dielectric interfaces, analogous to what occurs in the Kretschmann configuration. The SPP2 pole plays a similar role to that of the SPP1 pole, but less intense because of the dispersion relation calculated at the gold/prism interface. Radiation fields terms also play a fundamental role in the field distribution which, in fact, is the composition of several terms with varying wavelengths that compose the total field, be it electric or magnetic, by the spectral representation; of course, some of these terms will contribute in a more significant way than others.

Note in Figure 16(a) that the intensity of the electric field of the SPP wave, near the gold layer, is higher than the radiation fields in $\mathrm{z}$ points far away from the gold layer.
By the relation (48) it is possible to carry out a preliminary analysis of the variation of the plasma angle depending on the relative permittivity in medium 1 , for the SPCE sensor coupled to a nanoparticle array with period $\Delta_{c}=100 \mathrm{~nm}$ (we made it clear that this is a preliminary analysis because it is not the current purpose of the work). Figure 17 shows the generalized transmission coefficient in terms of the coupling angle.

At first, we can verify that, with the increase of refractivity index in medium 1, we have a displacement of the plasmonic angle in the $90^{\circ}$ direction and decay of the transmitted signal. For this reason, we can assume that these will be the changes in the optical response of the sensor.

\section{Conclusions}

In this work a theoretical electromagnetic model was presented for a SPR sensor in the SPCE configuration, coupled with a periodic planar array of equivalent nanodipoles. The Periodic Green's Function method was applied 


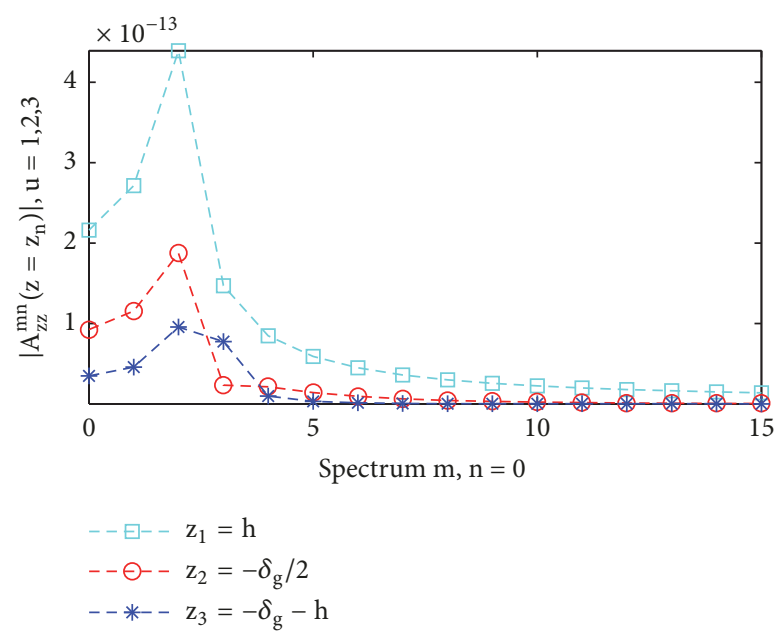

(a)

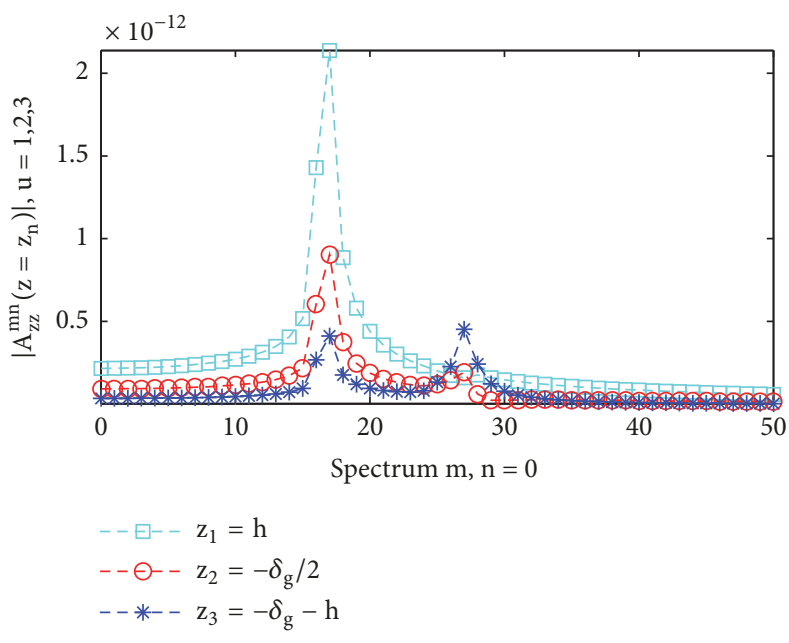

(b)

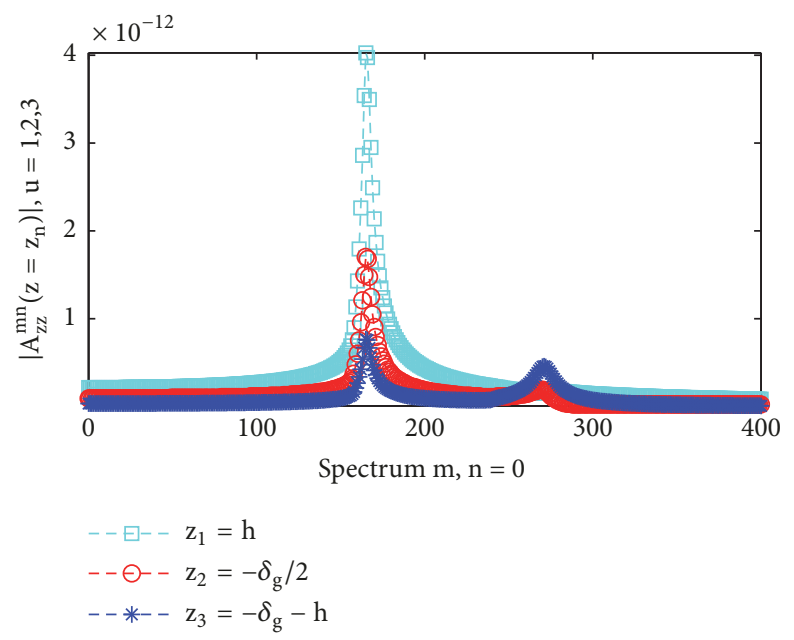

(c)

Figure 14: Spectral Coefficient $A_{z z}^{m n}(z)$ at $z=z_{u}$, with $u=1,2,3$ and $n=0$, for (a) $\Delta_{c}=1 \mu m$; (b) $\Delta_{c}=10 \mu m$; (c) $\Delta_{c}=100 \mu m$. In these results we set the following: $\delta_{g}=50 \mathrm{~nm}, h=20 \mathrm{~nm}, \theta^{\prime}=\phi^{\prime}=0^{\circ}$, and $\lambda=632.8 \mathrm{~nm}$.

to the magnetic potential, where the spectral representation method of the Fourier Double Complex Series was used.

From the magnetic potential field results, we find that $\delta_{g}=50 \mathrm{~nm}$ has been the ideal thickness of the gold layer in the sensor structure for the operating frequency. The electric and magnetic field results showed that the excitation of SPP waves, such as the coupling of highly polarized TM waves, arises most efficiently when the equivalent dipoles are oriented at $\theta^{\prime}=0^{\circ}$ (VED). In the case of the relative distance between the array and the surface of the sensor, defined by the chemical spacer, no major changes in the results were observed for the variation of parameter $h$, only simple changes of intensity in the prism region.

In the spectral analysis, we verified the emergence of the SPP poles in the spectral domain $m n$, for three cell periods. Also, we presented a convergence analysis of the series, where the terms of greater contribution in the spectral representation of PGF were identified. Despite the appearance of the SPP2 pole, at the gold/prism interface, the SPP1 pole was shown to contribute predominantly to the spectral representation.

As the period of the analysis cell increases, the results approach the limiting case, where there are no interactions between the nanoparticles in the array. The convergence of the method depends strongly on the cell period, being faster for relatively smaller cells and becoming slow as the cell period increased. Thus, we see the need for a greater number of terms, in the convergence of the method, for relatively larger cell periods. In this situation, we have the transition from the discrete spectrum to the continuous spectrum, when the summation in the spectral representation becomes an integral. However, the transition from discrete to continuous spectrum still encounters some difficulties, since in the discrete spectrum the propagation constant $k_{\rho}$ is purely real; on the other hand, in the continuous spectrum 


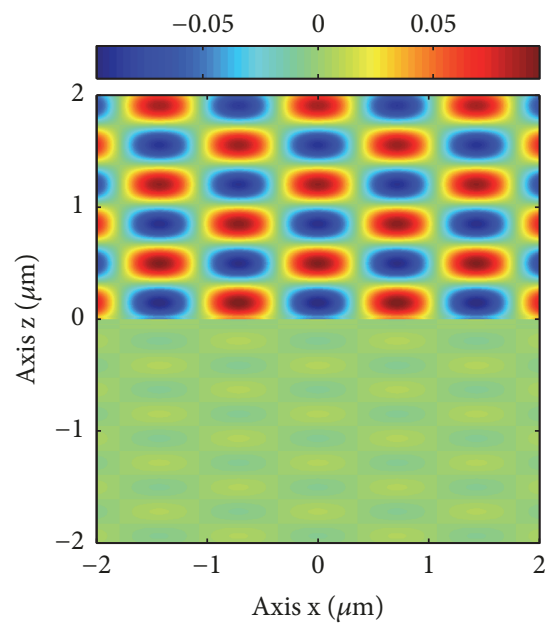

(a)

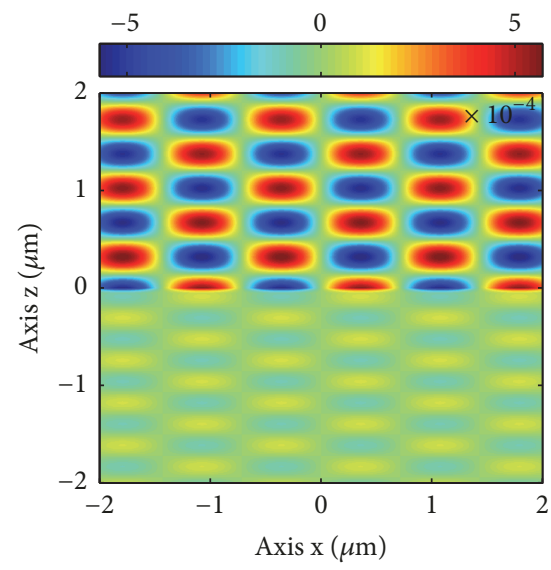

(d)

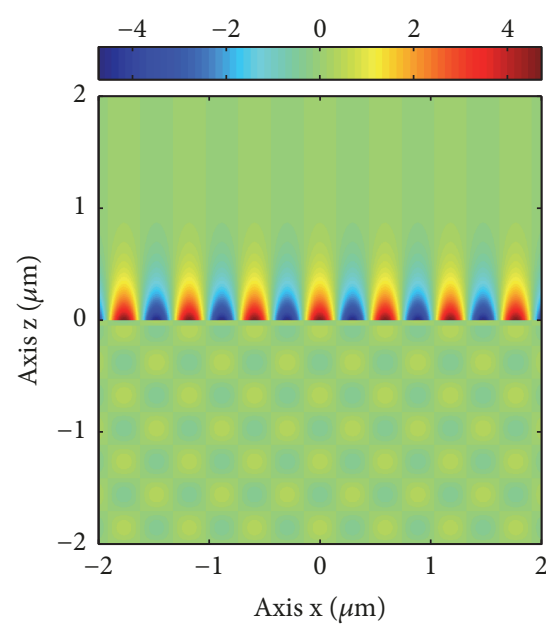

(b)

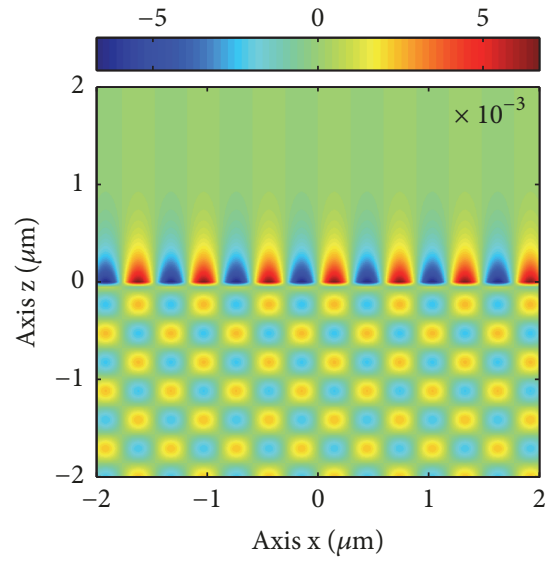

(e)

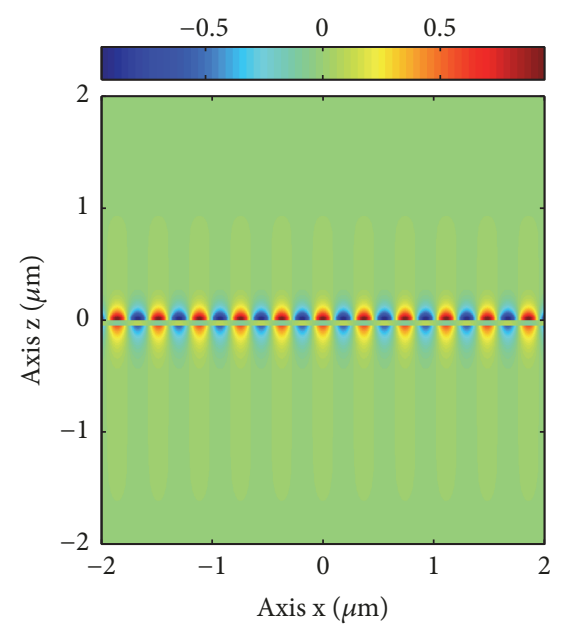

(c)

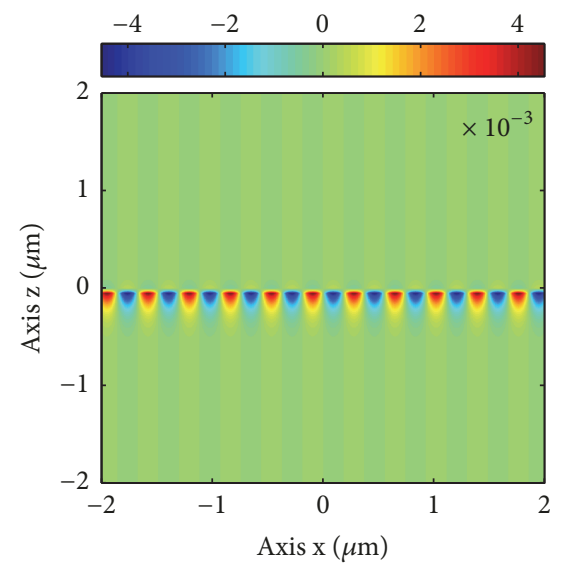

(f)

Figure 15: $E_{z}$ and $H_{y}$ in the $x z$ plane, at $y=0$, with $\Delta_{c}=10 \mu m$. $\operatorname{Re}\left\{E_{z}\right\}$ for the term $n=0$ and (a) $m=7$, (b) $m=m_{S P P 1}=17$, and (c) $m=m_{S P P 2}=27 ; \operatorname{Re}\left\{H_{y}\right\}$ for the term $n=0$ and (d) $m=7$, (e) $m=m_{S P P 1}=17$, and (f) $m=m_{S P P 2}=27$. In these results we set the following: $\delta_{g}=50 \mathrm{~nm}, h=20 \mathrm{~nm}, \theta^{\prime}=\phi^{\prime}=0^{\circ}$, and $\lambda=632.8 \mathrm{~nm}$.

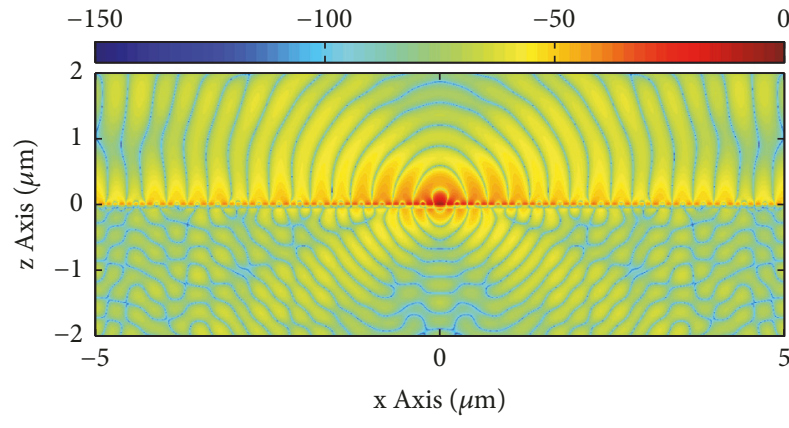

(a)

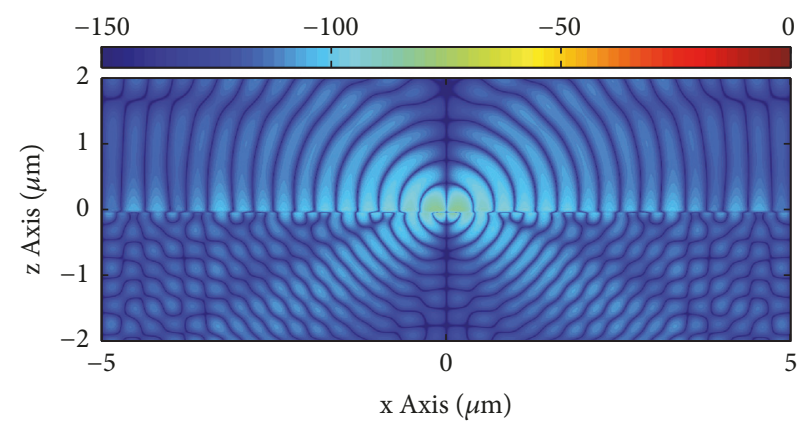

(b)

FIGURE 16: Electric and Magnetic Fields in the $x z$ plane, at $y=0$, with $\Delta_{c}=10 \mu m$ : (a) $20 \log \left|\operatorname{Re}\left\{E_{z}\right\}\right|$; (b) $20 \log \left|\operatorname{Re}\left\{H_{y}\right\}\right|$. In these results we set the following: $\delta_{g}=50 \mathrm{~nm}, h=20 \mathrm{~nm}, \theta^{\prime}=\phi^{\prime}=0^{\circ}$, and $\lambda=632.8 \mathrm{~nm}$.

$k_{\rho}$ presents a small imaginary part, as verified by [10]. This small imaginary part can be seen as the losses in the material.

The field results showed consistency with the observation of polarized TM waves, with high directivity in the prism region, which in the far field should form the characteristic of the sensor output, the light cone. In general, the results obtained showed good agreement, both mathematical and physical, demonstrating that the PGF method is efficient and 


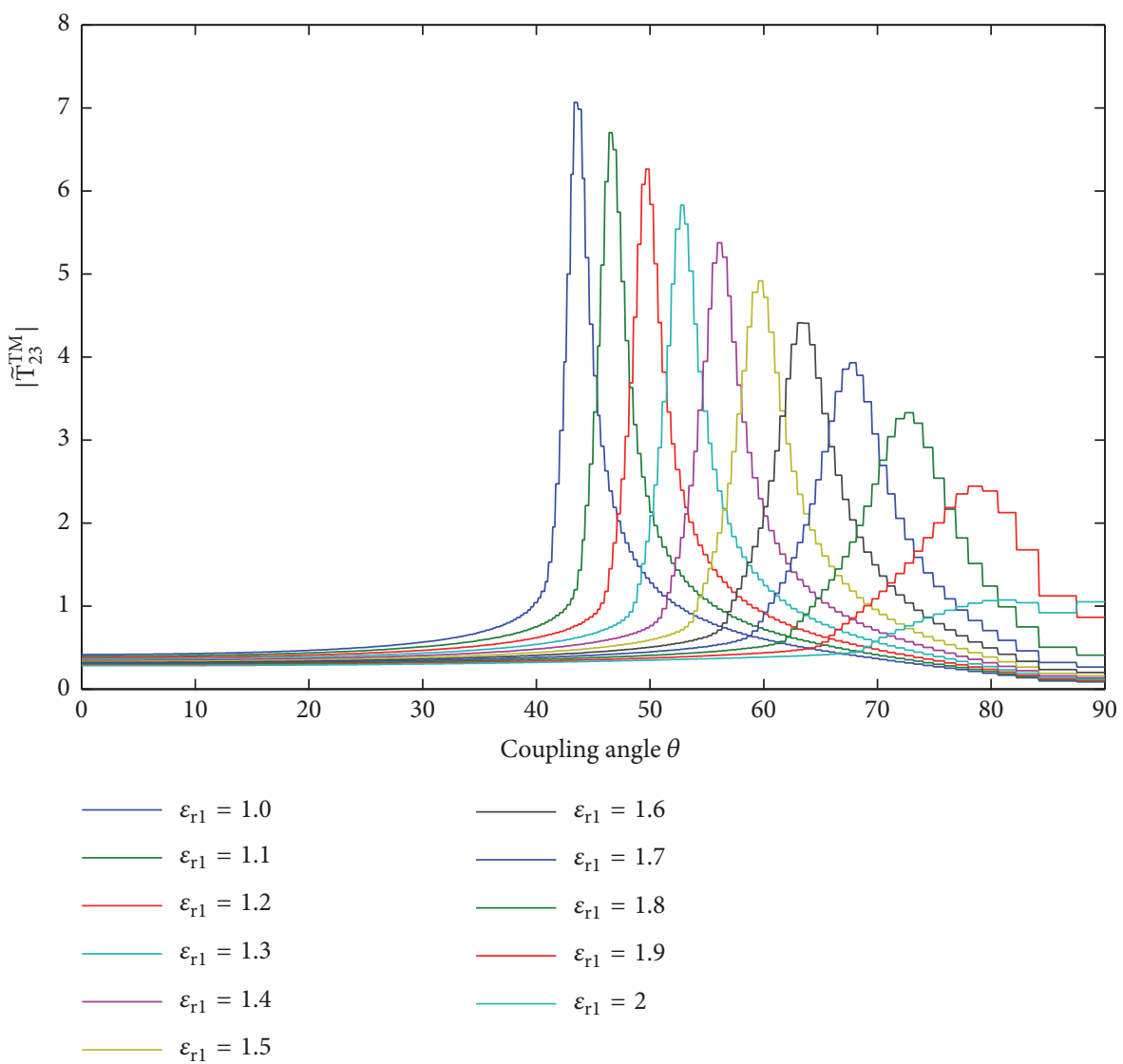

FIGURE 17: Generalized transmission coefficient in the gold/prism interface as a function of the coupling angle $\theta$.

can be used as a tool in the design and optimization of the sensor in the SPCE configuration.

For future work, we propose to verify the possibility of far field calculation from the obtained electromagnetic model, thus verifying the directivity and intensity of far field in function of the refractive characteristics of the sample. In addition, we will verify the temporal decay characteristics of the radiation emitted by the fluorophores and the total response of the sensor excited by a plane wave.

\section{Abbreviations}

The following abbreviations are used in this paper:

SPR: $\quad$ Surface Plasmon Resonance

RSPL: Localized Surface Plasmon Resonance

SPP: $\quad$ Surface Plasmon Polaritons

PGF: Periodic Green's Function

SPCE: Surface Plasmon Coupled Emission

AuNps: Gold Nanoparticles

DCIM: Discrete Complex Images Method

SW: $\quad$ Surface Wave.

\section{Data Availability}

The data used to support the findings of this study are available from the corresponding author upon request.

\section{Conflicts of Interest}

The authors declare no conflicts of interest.

\section{Acknowledgments}

Thanks are due to the members of the Nanophotonics and Nanoelectronics Laboratory from UFPA, Nanotribo. National Council for Scientific and Technological Development - CNPq (grant numbers: 423614/2018-5).

\section{References}

[1] D. W. Pohl, A. Alu, N. Engheta, and F. Marquier, Optical Antennas, Forthcoming Publications: Science, PRL, 2013.

[2] J. Homola, Surface Plasmon Resonance Based Sensors, Springer Series on Chemical Sensors and Biosensors, Springer, Berlin, Germany, 2006.

[3] S. A. Maier, Plasmonics: Fundamentals and Applications, Springer, NewYork, NY, USA, 1st edition, 2007.

[4] K. Q. Da Costa, J. S. Costa, V. Dmitriev, T. Del Rosso, O. Pandoli, and R. Q. Aucelio, "Analysis of surface plasmon resonance sensor coupled to periodic array of gold nanoparticles," in Proceedings of the SBMO/IEEE MTT-S International Microwave and Optoelectronics Conference, IMOC 2015, pp. 1-5, Brazil, November 2015.

[5] A. Cruz and K. Costa, "Extinction efficiency and electromagnetic fields of isolated and coupled core-shell nanoparticles," 
Journal of Communication and Information Systems, vol. 33, no. 1, 2018.

[6] R. Marani, M. Grande, V. Petruzelli, and A. D’Orazio, "Plasmonic bandgaps in $1 \mathrm{D}$ arrays of slits on metal layers excited by out-of-plane sources," International Journal of Optics, vol. 2012, Article ID 146396, 12 pages, 2012.

[7] T. Špringer, M. L. Ermini, B. Špačková, J. Jabloňků, and J. Homola, "Enhancing sensitivity of surface plasmon resonance biosensors by functionalized gold nanoparticles: size matters," Analytical Chemistry, vol. 86, no. 20, pp. 10350-10356, 2014.

[8] M. A. Cooper, "Optical biosensors in drug discovery," Nature Reviews Drug Discovery, vol. 1, no. 7, pp. 515-528, 2002.

[9] J. Malicka, I. Gryczynski, Z. Gryczynski, and J. R. Lakowicz, "Use of surface plasmon-coupled emission to measure DNA hybridization," Journal of Biomolecular Screening, vol. 9, no. 3, pp. 208-215, 2004.

[10] N. W. P. Souza, Análise de Sensores Plasmônicos Baseados em Nano Estruturas Estratificadas Utilizando o Método das Imagens Complexas Discretas [Doctoral thesis], PPGEE, UFPA, BelémPA, Brazil, 2018.

[11] N. W. P. de Souza, J. S. Costa, R. C. Santos, A. F. S. da Cruz, T. D. Rosso, and K. Q. da Costa, "Modal analysis of surface plasmon resonance sensor coupled to periodic array of coreshell metallic nanoparticles," in Resonance, J. Awrejcewicz, Ed., InTech, 2017.

[12] A. F. S. Cruz, N. W. P. de Souza, V. Dmitriev, and K. Q. Costa, "Modelo Eletromagnético de Sensor Plasmônico Acoplado a Arranjo de Nanopartículas via PGF 3D," in MOMAG 2018, 2018, Santa Rita do Sapucaí - MG. $13^{\circ} \mathrm{CBMag}$ - Congresso Brasileiro de Eletromagnetismo $18^{\check{z}}$ SBMO - Simpósio Brasileiro de Microondas e Optoeletrônica INATEL, 2018, 2018.

[13] T. Del Rosso, Q. Zaman, M. Cremona, O. Pandoli, and A. R. J. Barreto, "SPR sensors for monitoring the degradation processes of Eu (dbm) 3 (phen) and Alq3 thin films under atmospheric and UVA exposure," Applied Surface Science, vol. 442, pp. 759766, 2018.

[14] A. F. Cruz, K. Q. Costa, V. Dmitriev, and T. Del Rosso, "Spectral analysis of a surface plasmon resonance sensor of nanoparticles by periodic green function," in Proceedings of the 2018 SBFoton International Optics and Photonics Conference (SBFoton IOPC), pp. 1-5, Campinas, Brazil, October 2018.

[15] D. Marsh, "Reaction fields in the environment of fluorescent probes: polarity profiles in membranes," Biophysical Journal, vol. 96, no. 7, pp. 2549-2558, 2009.

[16] D. G. Dudley, Mathematical Foundations for Electromagnetic Theory, IEEE press, New York, NY, USA, 1994.

[17] W. C. Chew, Waves and Fields in Inhomogeneous Media, IEEE press, 1995.

[18] C. A. Balanis, Advanced Engineering Electromagnetics, John Wiley \& Sons, 1999.

[19] P. H. Chung, C. Tregidgo, and K. Suhling, "Determining a fluorophore's transition dipole moment from fluorescence lifetime measurements in solvents of varying refractive index," Methods and Applications in Fluorescence, vol. 4, no. 4, Article ID 045001, 2016. 


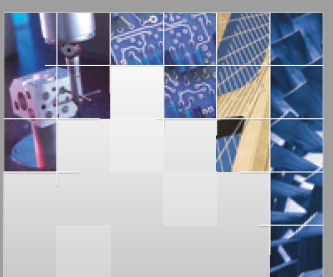

\section{Enfincering}
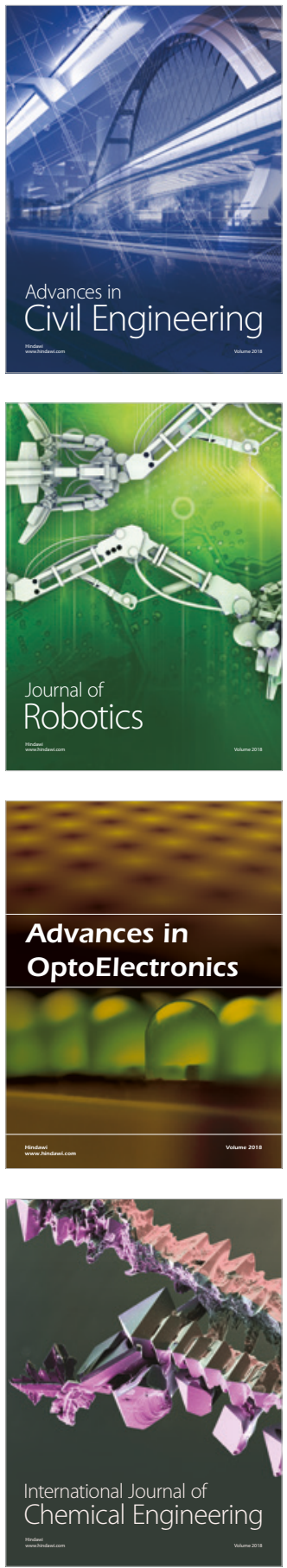

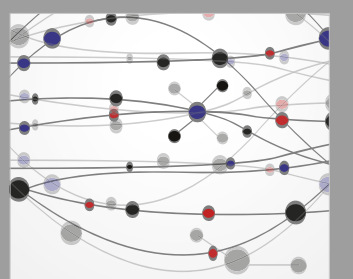

\section{Rotating \\ Machinery}

The Scientific World Journal

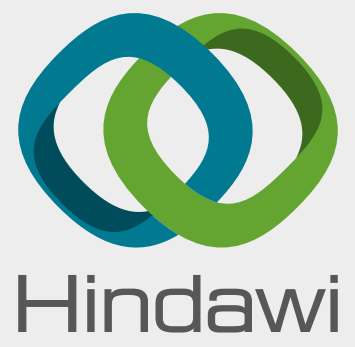

Submit your manuscripts at

www.hindawi.com
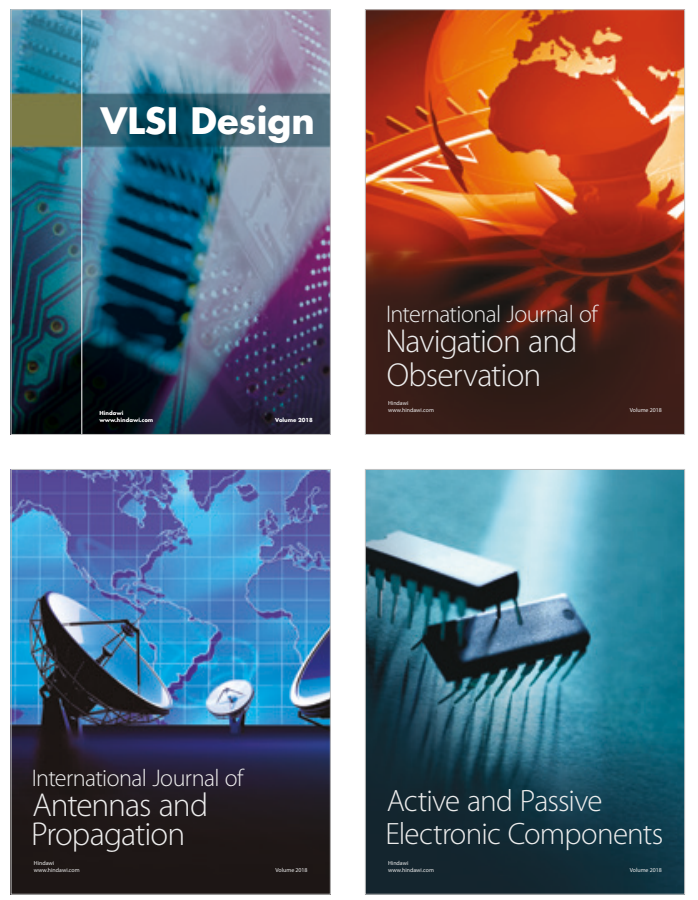
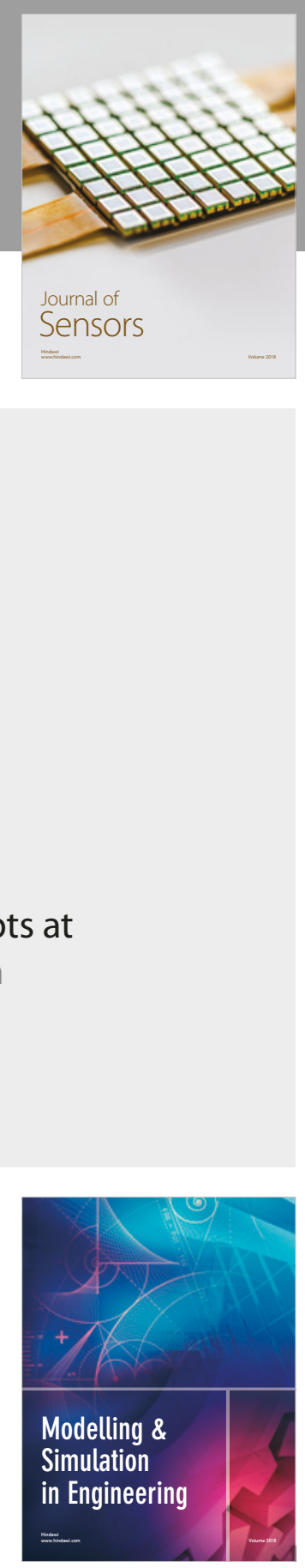

\section{Advances \\ Multimedia}
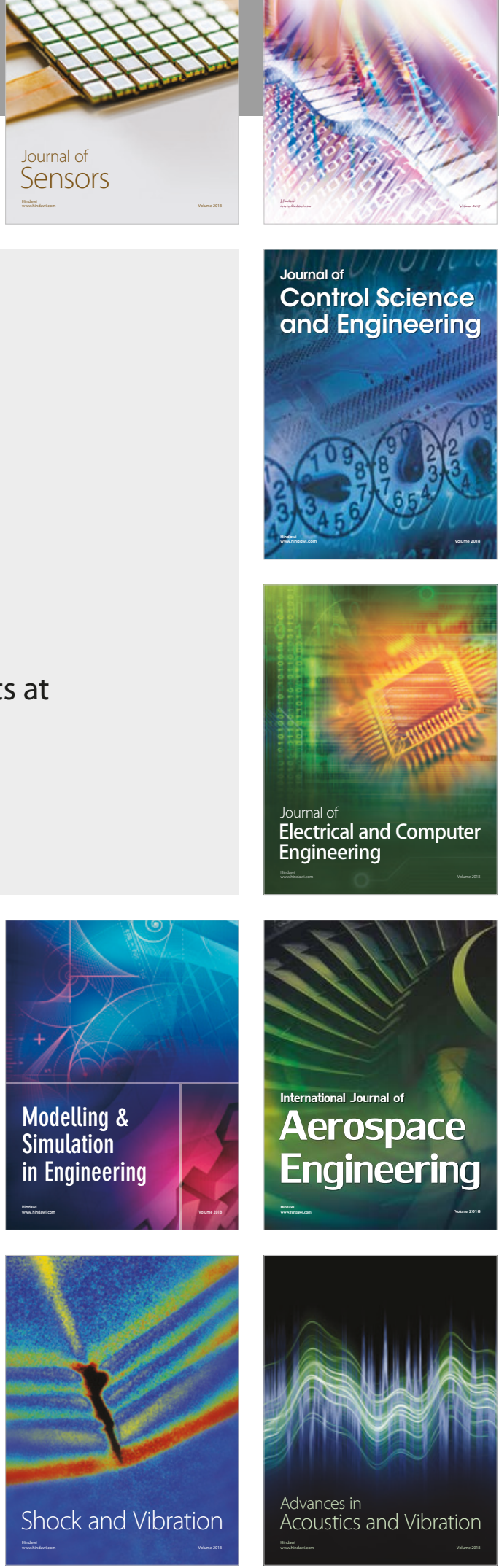